\title{
Systematic model reduction captures the dynamics of extrinsic noise in biochemical subnetworks
}

Cite as: J. Chem. Phys. 153, 025101 (2020); https://doi.org/10.1063/5.0008304

Submitted: 20 March 2020 . Accepted: 22 June 2020 . Published Online: 09 July 2020

Barbara Bravi (D), Katy J. Rubin, and Peter Sollich (i)
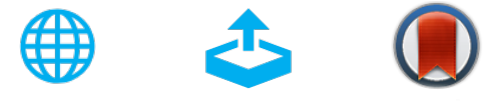

\section{ARTICLES YOU MAY BE INTERESTED IN}

Optimal estimates of self-diffusion coefficients from molecular dynamics simulations The Journal of Chemical Physics 153, 024116 (2020); https:// doi.org/10.1063/5.0008312

Velocity jump processes: An alternative to multi-timestep methods for faster and accurate molecular dynamics simulations

The Journal of Chemical Physics 153, 024101 (2020); https://doi.org/10.1063/5.0005060

Efficient and exact sampling of transition path ensembles on Markovian networks

The Journal of Chemical Physics 153, 024121 (2020); https://doi.org/10.1063/5.0012128

\section{Lock-in Amplifiers up to $600 \mathrm{MHz}$}
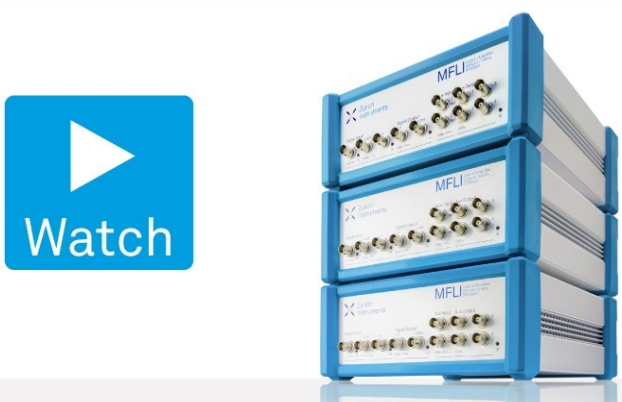


\title{
Systematic model reduction captures the dynamics of extrinsic noise in biochemical subnetworks
}

\author{
Cite as: J. Chem. Phys. 153, 025101 (2020); doi: 10.1063/5.0008304 \\ Submitted: 20 March 2020 - Accepted: 22 June 2020 • \\ Published Online: 9 July 2020
}

Barbara Bravi, $^{1, a)}$ (D) Katy J. Rubin, ${ }^{2}$ and Peter Sollich ${ }^{2,3, b)}$ (D)

\begin{abstract}
AFFILIATIONS
${ }^{1}$ Institute of Theoretical Physics, Ecole Polytechnique Fédérale de Lausanne (EPFL), CH-1015 Lausanne, Switzerland

²Department of Mathematics, King's College London, Strand, London WC2R 2LS, United Kingdom

${ }^{3}$ Institute for Theoretical Physics, Georg-August-University Göttingen, Friedrich-Hund-Platz 1, 37077 Göttingen, Germany
\end{abstract}

\author{
a) Author to whom correspondence should be addressed: barbarabravi@ymail.com. \\ Current address: Laboratoire de Physique de l'Ecole Normale Supérieure, ENS, Université PSL, CNRS, \\ Sorbonne Université, Université de Paris, F-75005 Paris, France. \\ b) Electronic mail: peter.sollich@uni-goettingen.de
}

\begin{abstract}
We consider the general problem of describing the dynamics of subnetworks of larger biochemical reaction networks, e.g., protein interaction networks involving complex formation and dissociation reactions. We propose the use of model reduction strategies to understand the "extrinsic" sources of stochasticity arising from the rest of the network. Our approaches are based on subnetwork dynamical equations derived by projection methods and path integrals. The results provide a principled derivation of different components of the extrinsic noise that is observed experimentally in cellular biochemical reactions, over and above the intrinsic noise from the stochasticity of biochemical events in the subnetwork. We explore several intermediate approximations to assess systematically the relative importance of different extrinsic noise components, including initial transients, long-time plateaus, temporal correlations, multiplicative noise terms, and nonlinear noise propagation. The best approximations achieve excellent accuracy in quantitative tests on a simple protein network and on the epidermal growth factor receptor signaling network.
\end{abstract}

Published under license by AIP Publishing. https://doi.org/10.1063/5.0008304

\section{INTRODUCTION}

Networks of biochemical reactions can comprise thousands of species, making mathematical modeling of their full structure a difficult task. The many parameters often cannot be estimated with the precision that would be required for reliable quantitative predictions. Focusing on a few, well-characterized species, i.e., "reducing" the model to a subnetwork, is then necessary for the sake of analysis and qualitative understanding. ${ }^{1-3}$ In this paper, we address the question of how noise should be incorporated into such reduced models. The complex kinetics of, e.g., intracellular biochemical networks is crucially determined by fluctuations, their interplay with nonlinearities giving rise to a rich set of dynamical behaviors such as signal amplification and damping, focusing of oscillations, ${ }^{4}$ and bimodal profiles of gene expression ${ }^{5}$ and cell responses. ${ }^{6}$ These effects can make noise either detrimental or functional to signal processing within cells. 'Accounting properly for fluctuations while retaining the benefits of reduced models thus becomes a key challenge; to address it, one has to keep track of the effects of the embedding environment-which we will call the "bulk"-of a chosen subnetwork. The resulting dynamical information will be crucial in identifying and ultimately measuring the different drivers of cellular heterogeneity. This would be of great benefit in synthetic biology applications that aim to design circuits where the effects of the biomolecular context are fully characterized and could even be exploited for specific functions.

Among possible approaches to model reduction, ${ }^{1-3,8,9}$ projection methods from statistical mechanics ${ }^{10,11,55,56}$ are attractive as they allow one to project a given dynamics systematically onto an arbitrary subspace of subnetwork observables. In protein-protein 
interaction networks, this has been shown to improve the accuracy of quantitative predictions for the subnetwork dynamics, ${ }^{12}$ but the method could track only averages over the intrinsic subnetwork noise, with fluctuations arising solely from the potentially random bulk initial conditions. An alternative field-theoretic approach for reduction to subnetwork dynamics avoids this restriction. ${ }^{13}$ This approximate method relies on perturbations to a Gaussian theory and so will be referred to as "third order corrected Gaussian Approximation" (3GA) henceforth. Its key advantage for our present purposes is that it can, in principle, account for all stochastic fluctuations including those from intrinsic subnetwork noise. In the limit of vanishing intrinsic noise, the two schemes become essentially identical: ${ }^{12,13}$ while they produce subnetwork dynamical equations with a different structure, one can prove ${ }^{13}$ that the resulting predictions agree up to quadratic order in deviations from a dynamical fixed point.

The presence of the surrounding bulk system generates both memory terms in the subnetwork dynamics as well as extrinsic noise, ${ }^{14,15}$ which is the focus of our interest here. Hence, as our first new contribution, we extend the formalism of previous studies ${ }^{12,13}$ to extrinsic noise stemming from two sources: the fact that the initial deviation from steady state in the bulk is unknown and therefore can be taken as randomly distributed, and the intrinsic stochastic fluctuations of the dynamics, captured only by the $3 \mathrm{GA}$ framework. Importantly, our results do not rely on any timescale separation, while projection methods and more recently path integrals have so far been applied primarily to coarsegraining by elimination of fast bulk variables. ${ }^{16-19}$ As a second novel contribution, we propose and analyze approximations of the extrinsic noise terms that help us evaluate them more expediently and elucidate the relative contribution of different noise properties such as color, non-linearity, and multiplicativity of the source.

The structure of this paper is as follows: We first recall key features of model reduction approaches for subnetwork dynamics in Sec. II, starting from the case of linear reaction equations to build insight (Sec. II B). We then derive the extrinsic noise terms for the case of nonlinear dynamics, separately by the projection method (Sec. II C) and 3GA (Sec. II D). In Sec. III, we assess the accuracy of subnetwork equations with stochastic terms arising only from uncertain bulk initial states for which a comparison between 3GA and projection methods can be made. As, in general, it will be complicated to estimate the full time-dependence of these stochastic terms from measurements on the bulk, we propose an approximation aimed at capturing the long and short time subnetwork behavior (Sec. III C). The restriction to negligible intrinsic noise can be easily removed in the $3 \mathrm{GA}$ approach, allowing us to characterize all the intrinsic and extrinsic terms that contribute to the subnetwork noise. We compare the 3GA performance to the steady state linear noise approximation ${ }^{16,20}$ and to intermediate approximations that neglect either nonlinearity or time correlations in the extrinsic noise (Sec. IV). In addition, we estimate how the accuracy of all these approximations scales with the strength of extrinsic noise in Secs. III D and IV B. For this detailed analysis, we use a simple network model; in Sec. V, we extend this and explore the application of different model reduction approaches to a paradigmatic signaling pathway, the reaction network around the Epidermal Growth Factor Receptor (EGFR).

\section{MODEL REDUCTION METHODS FOR SUBNETWORK DYNAMICS}

\section{A. Subnetworks in protein-protein interaction networks}

We consider a reaction network of $N$ molecular species such as proteins and protein complexes, with concentrations $\boldsymbol{x}=\left(x_{1}\right.$, ..., $\left.x_{N}\right)$, evolving in time according to a set of Chemical Langevin Equations (CLEs), ${ }^{21,22}$

$$
\frac{d x_{i}(t)}{d t}=[\boldsymbol{S} \boldsymbol{f}(\boldsymbol{x}(t))]_{i}+\xi_{i}(t), \quad i=1, \ldots, N .
$$

In the deterministic part $\boldsymbol{S} \boldsymbol{f}, \boldsymbol{S}$ denotes the stoichiometric matrix of size $N \times R$ ( $R$ being the number of chemical reactions) and $\boldsymbol{f}$ is the $R$-dimensional vector of reaction fluxes. The elements of $\boldsymbol{S} \boldsymbol{f}$ read

$$
\begin{aligned}
{[\boldsymbol{S f}(\boldsymbol{x}(t))]_{i}=} & \sum_{j \neq l}\left(k_{l, i j}^{-} x_{i}(t)-k_{i j, l}^{+} x_{i}(t) x_{j}(t)\right) \\
& +\frac{1}{2} \sum_{j \neq l}\left(k_{j l, i}^{+} x_{j}(t) x_{l}(t)-k_{i, j l}^{-} x_{i}(t)\right) \\
& +\sum_{j}\left(\lambda_{j i} x_{j}(t)-\lambda_{i j} x_{i}(t)\right) \\
& +\sum_{l}\left(2 k_{l, i i}^{-} x_{l}(t)-k_{i i, l}^{+} x_{i}(t) x_{i}(t)\right) \\
& +\sum_{j}\left(\frac{1}{2} k_{j j, i}^{+} x_{j}(t) x_{j}(t)-k_{i, j j}^{-} x_{i}(t)\right)
\end{aligned}
$$

and captures binary reactions, such as complex formation (with rate constants $k_{i j, l}^{+}, k_{j l, i}^{+}, k_{i i, l}^{+}$, and $\left.k_{j j, i}^{+}\right)$and dissociation $\left(k_{l, i j}^{-}, k_{i, j l}^{-}, k_{l, i i}^{-}\right.$, and $\left.k_{i, j j}^{-}\right)$, as well as the unary reactions $\left(\lambda_{i j}\right.$ and $\left.\lambda_{j i}\right)$, e.g., phosphorylation or conformational change. The final term $\xi_{i}(t)$ in (1) is an intrinsic noise term from the stochasticity of reaction events, which is Gaussian distributed with zero mean and covariance

$$
\left\langle\xi_{i}(t) \xi_{j}\left(t^{\prime}\right)\right\rangle=\boldsymbol{\Sigma}_{i j}(x(t)) \delta\left(t-t^{\prime}\right), \quad \boldsymbol{\Sigma}(\boldsymbol{x}(t))=\varepsilon \boldsymbol{S} \operatorname{diag}(\boldsymbol{f}(\boldsymbol{x}(t))) \boldsymbol{S}^{\mathrm{T}} .
$$

The $\boldsymbol{x}$-dependent correlations make this noise multiplicative to be interpreted here in the Itô convention, ${ }^{23}$ while the temporal correlations remain white. The scale of the noise variance is set by the inverse reaction volume ${ }^{22} \varepsilon=1 / V$, implying that fluctuations become less important for large reaction volumes $V$ but have a nonnegligible effect on reactions occurring in cellular subcompartments that involve small to moderate copy numbers.

We assume that only a small subnetwork of species is well-characterized (e.g., because their biochemical interactions are known or their time evolution can be measured experimentally with high precision), while the rest of the network (the bulk) is not. In this paper, we address the problem of deriving an accurate description of the dynamics of the chosen subnetwork that also incorporates the dynamical effects of extrinsic noise due to the embedding into the bulk environment.

An important aspect of our treatment is that the choice of subnetwork is not driven by the model reduction procedure itself but, in principle, arbitrary. To make explicit expressions shorter, we simplify the subnetwork equations by making specific plausible assumptions about the split into subnetwork and bulk: given 
that bulk species are assumed uninteresting or poorly characterized, complexes involving at least one bulk species are themselves assigned to the bulk, while complexes made up of two subnetwork species are assigned to the subnetwork.

\section{B. Subnetwork modeling with a linearized Langevin dynamics}

We first look at the case of linearized dynamics, which can be treated by direct elimination of bulk variables. The subnetwork reduced dynamics found in this case is exact (with respect to the already linearized dynamics) and gives useful insights into the structure that emerges from integrating out the bulk. Let us rewrite concentrations as $\boldsymbol{x}(t)=\boldsymbol{y}+\delta \boldsymbol{x}(t)$, where $\delta \boldsymbol{x}(t)=\boldsymbol{x}(t)-\boldsymbol{y}$ are deviations from the steady state $y$, which is found from the deterministic part of the dynamics by solving $\boldsymbol{S} \boldsymbol{f}(\boldsymbol{y})=0$. We rewrite (1) in terms of $\delta \boldsymbol{x}(t)$, and by retaining only terms linear in $\delta \boldsymbol{x}(t)$, one obtains the dynamics linearized about the steady state

$$
\frac{d}{d t} \delta \boldsymbol{x}^{\mathrm{T}}(t)=\delta \boldsymbol{x}^{\mathrm{T}}(t) \boldsymbol{L}+\boldsymbol{\xi}_{0}^{\mathrm{T}}(t)
$$

where $\mathrm{T}$ denotes the transpose of a column vector. The effective rate matrix $L$ is defined as

$$
\boldsymbol{L}_{j i}=\left.\frac{\partial}{\partial x_{j}}[\boldsymbol{S} \boldsymbol{f}(\boldsymbol{x})]_{i}\right|_{x=y} .
$$

The white Gaussian noise $\boldsymbol{\xi}_{0}(t)$ appearing in the linearized dynamics is additive; we will use the subscript 0 to indicate this property. Its time correlations are

$$
\left\langle\boldsymbol{\xi}_{0}(t) \xi_{0}^{\mathrm{T}}\left(t^{\prime}\right)\right\rangle=\Sigma_{0} \delta\left(t-t^{\prime}\right)
$$

with a covariance matrix $\boldsymbol{\Sigma}_{0}=\boldsymbol{\Sigma}(\boldsymbol{x}(t)=\boldsymbol{y})$ that is constant in time and equal to the one obtained by performing a linear noise approximation $^{24}$ around the steady state $\boldsymbol{y}$. Our linearization is in exactly the same spirit as the latter approximation, i.e., we keep all terms in an expansion around the steady state that can be included while retaining purely Gaussian fluctuations.

As we are interested in modeling a subnetwork of species, we separate the $N$-dimensional vector of concentration deviations $\delta \boldsymbol{x}(t)$ into a subnetwork part, $\delta x^{\mathrm{s}}(t)$, and a bulk part, $\delta x^{\mathrm{b}}(t)$. It is convenient to choose a numbering where $i=1, \ldots, N^{\mathrm{s}}$ labels subnetwork species, while the remaining $N^{\mathrm{b}}=N-N^{\mathrm{s}}$ species $i=N^{\mathrm{s}}+1$, $\ldots, N$ belong to the bulk, giving for the rate matrix (5) the block decomposition

$$
\boldsymbol{L}=\left(\begin{array}{l}
\boldsymbol{L}^{\mathrm{ss}} \boldsymbol{L}^{\mathrm{bs}} \\
\boldsymbol{L}^{\mathrm{sb}} \boldsymbol{L}^{\mathrm{bb}}
\end{array}\right)
$$

Equation (4) can then be split into a set of equations for the subnetwork and one for the bulk

$$
\begin{aligned}
& \frac{d}{d t} \delta \boldsymbol{x}^{\mathrm{s}^{\mathrm{T}}}=\delta \boldsymbol{x}^{\mathrm{s}^{\mathrm{T}}} \boldsymbol{L}^{\mathrm{ss}}+\delta \boldsymbol{x}^{\mathrm{b}^{\mathrm{T}}} \boldsymbol{L}^{\mathrm{bs}}+\boldsymbol{\xi}_{0}^{s^{\mathrm{T}}}, \\
& \frac{d}{d t} \delta \boldsymbol{x}^{\mathrm{b}^{\mathrm{T}}}=\delta \boldsymbol{x}^{\mathrm{b}^{\mathrm{T}}} \boldsymbol{L}^{\mathrm{bb}}+\delta \boldsymbol{x}^{\mathrm{s}^{\mathrm{T}}} \boldsymbol{L}^{\mathrm{sb}}+\boldsymbol{\xi}_{0}^{\mathrm{b}^{\mathrm{T}}},
\end{aligned}
$$

where the noise correlations are given by the block form of the diffusion matrix

$$
\Sigma_{0}=\left(\begin{array}{l}
\Sigma_{0}^{\mathrm{ss}} \Sigma_{0}^{\mathrm{sb}} \\
\Sigma_{0}^{\mathrm{bs}} \Sigma_{0}^{\mathrm{bb}}
\end{array}\right), \quad \boldsymbol{\Sigma}_{0}^{\mathrm{bs}}=\left(\boldsymbol{\Sigma}_{0}^{\mathrm{sb}}\right)^{\mathrm{T}} .
$$

The reduction to subnetwork dynamics can now be achieved by direct elimination of the bulk degrees of freedom (DOF): we first solve explicitly for $\delta x^{\mathrm{b}}$

$\delta \boldsymbol{x}^{\mathrm{b}^{\mathrm{T}}}(t)=\delta \boldsymbol{x}^{\mathrm{b}^{\mathrm{T}}}(0) e^{L^{\mathrm{bb}} t}+\int_{0}^{t} d t^{\prime}\left(\delta \boldsymbol{x}^{\mathrm{s}^{\mathrm{T}}}\left(t^{\prime}\right) \boldsymbol{L}^{\mathrm{sb}}+\xi_{0}^{\mathrm{b}^{\mathrm{T}}}\left(t^{\prime}\right)\right) e^{L^{\mathrm{bb}}\left(t-t^{\prime}\right)}$

and then substitute into (7) to obtain the reduced subnetwork dynamics

$$
\frac{d}{d t} \delta \boldsymbol{x}^{\mathrm{s}^{\mathrm{T}}}(t)=\delta \boldsymbol{x}^{\mathrm{s}^{\mathrm{T}}}(t) \boldsymbol{L}^{\mathrm{ss}}+\int_{0}^{t} d t^{\prime} \delta \boldsymbol{x}^{\mathrm{s}^{\mathrm{T}}}\left(t^{\prime}\right) \boldsymbol{M}^{\mathrm{ss}}\left(t-t^{\prime}\right)+\chi_{0}^{\mathrm{T}}(t),
$$

with

$$
\begin{gathered}
\boldsymbol{M}^{\mathrm{ss}}\left(t-t^{\prime}\right)=\boldsymbol{L}^{\mathrm{sb}} e^{L^{\mathrm{bb}}\left(t-t^{\prime}\right)} \boldsymbol{L}^{\mathrm{bs}} \\
\chi_{0}^{\mathrm{T}}(t)=\xi_{0}^{\mathrm{s}^{\mathrm{T}}}(t)+\int_{0}^{t} d t^{\prime} \boldsymbol{\xi}_{0}^{\mathrm{b}^{\mathrm{T}}}\left(t^{\prime}\right) e^{\boldsymbol{L}^{\mathrm{bb}}\left(t-t^{\prime}\right)} \boldsymbol{L}^{\mathrm{bs}}+\delta \boldsymbol{x}^{\mathrm{b}^{\mathrm{T}}}(0) e^{L^{\mathrm{bb}} t} \boldsymbol{L}^{\mathrm{bs}} .
\end{gathered}
$$

In (10), reactions occurring within the subnetwork appear in their original form via the subnetwork matrix $\boldsymbol{L}^{\text {ss }}$ while the embedding environment (the bulk) introduces non-Markovian terms. A memory function (11) appears because bulk DOF are influenced by the past behavior of the subnetwork and feed this influence back to the subnetwork at a later time. On the other hand, a colored (i.e., timecorrelated) noise (12) accounts for the intrinsic subnetwork noise $\xi_{0}^{\mathrm{s}}(t)$, for the effect of bulk intrinsic noise $\xi_{0}^{\mathrm{b}}\left(t^{\prime}\right)$ at a previous time $t^{\prime}$ on the subnetwork at time $t$, and for the (potentially unknown) initial deviations from steady state of the bulk, $\delta x^{b}(0)$. The two model reduction strategies introduced in Refs. 12 and 13, the first based on projection methods ${ }^{10,11}$ and the second based on a path integral representation of the stochastic dynamics, ${ }^{25}$ give the exact memory function (11). The projection approach averages over stochastic noise and so only retrieves the last term of (12). Importantly, both methods allow us to go beyond the linearized dynamics while still accounting explicitly for memory and extrinsic noise: we will refer to the latter as random force in the projection methods and colored noise in the path integral approach 3GA.

\section{Random force in projection methods}

The application of projection methods to networks of unary and binary protein reactions as in (1) was studied in Ref. 12 with a focus on memory terms. We next summarize those results and give the corresponding ones for the random force that interests us here.

The basic idea of projection methods is to define two operators projecting onto the subspace of subnetwork DOF and onto the orthogonal one, respectively. For nonlinear projected equations, even though a subnetwork has been fixed, a choice remains as to which set of subnetwork observables to project onto. We argued in Ref. 12 that the most convenient set consists of linear and quadratic observables, i.e., concentration deviations and their products. With this set fixed, one can find a matrix representation of the projection 
operators involved that is accurate up to corrections of order $\delta x^{3}$ (cubic in deviations from steady state) and $\mathscr{O}(\varepsilon)$ (linear in the noise variance). Therefore, the projected nonlinear equations will be exact up to $O\left(\delta x^{2}\right)$ and in the limit $\varepsilon \rightarrow 0$. We use the equations as derived for $\varepsilon \rightarrow 0$ but will see later that at least for the mean behavior, they can also give reasonable predictions when $\varepsilon>0$. Referring to Ref. 12 for details and to Sec. I of the supplementary material for a summary, the resulting projected equations read

$$
\begin{aligned}
\frac{d}{d t} \delta \boldsymbol{x}^{\mathrm{s}^{\mathrm{T}}}(t)= & \delta \boldsymbol{x}^{\mathrm{s}^{\mathrm{T}}}(t) \boldsymbol{L}^{\mathrm{ss}}+\left(\delta \boldsymbol{x}^{\mathrm{s}}(t) \circ \delta \boldsymbol{x}^{\mathrm{s}}(t)\right)^{\mathrm{T}} \boldsymbol{L}^{\mathrm{ss}, \mathrm{s}} \\
& +\int_{0}^{t} d t^{\prime} \delta \boldsymbol{x}^{\mathrm{s}^{\mathrm{T}}}\left(t^{\prime}\right) \boldsymbol{M}^{\mathrm{ss}}\left(t-t^{\prime}\right)+\int_{0}^{t} d t^{\prime}\left(\delta \boldsymbol{x}^{\mathrm{s}}\left(t^{\prime}\right) \circ \delta \boldsymbol{x}^{\mathrm{s}}\left(t^{\prime}\right)\right)^{\mathrm{T}} \\
& \times \boldsymbol{M}_{\mathrm{P}}^{\mathrm{ss}, \mathrm{s}}\left(t-t^{\prime}\right)+\boldsymbol{r}^{\mathrm{T}}(t)
\end{aligned}
$$

and generalize the linear reduced dynamics (10) to products of concentrations (and thus to nonlinear reactions such as formation of a complex from two other species). Here, $\boldsymbol{M}_{\mathrm{P}}^{\mathrm{ss} s}$ denotes the nonlinear memory from the projection approach [see Eq. (4) of the supplementary material]. Formally, the projected equations describe averages over the noise of the subnetwork concentrations conditional on specified initial values. We do not use separate nomenclature for these averages because they coincide with the concentration themselves in the noiseless limit considered here. We have introduced the notation $\boldsymbol{a} \circ \boldsymbol{b}$, not to be confused with the Hadamard (elementwise) product or the more similar Kronecker (outer) product, to denote the outer product $\boldsymbol{a} \boldsymbol{b}^{\mathrm{T}}$ rearranged into a single (column) vector. The entries of this vector are all possible componentwise products $a_{i} b_{j}$ so that, e.g., $\delta x^{\mathrm{s}} \circ \delta x^{\mathrm{b}}$ has dimension $N^{\mathrm{s}} N^{\mathrm{b}}$. Where the two vectors belong to the same subset (both " $\mathrm{s}$ " or both " $\mathrm{b}$ "), we mean the ordered products only: $\delta x^{s} \circ \delta x^{s}$ is the vector containing the $N^{s}\left(N^{s}+1\right) / 2$ products $\delta x_{i} \delta x_{j}$ with $1 \leq i \leq j \leq N^{s}$. The matrix $L^{s s, s}$ is a block of a larger $L$ matrix representing the full dynamics of all linear and quadratic observables. $L^{s s, s}$ contains the reaction rates in such a way to give

$$
\begin{aligned}
{\left[\left(\delta x^{\mathrm{s}} \circ \delta x^{\mathrm{s}}\right)^{\mathrm{T}} \boldsymbol{L}^{\mathrm{ss}, \mathrm{s}}\right]_{i}=} & \sum_{j, l=1, j \neq l}^{N^{\mathrm{s}}}\left(\frac{1}{2} k_{j l, i}^{+} \delta x_{j} \delta x_{l}-k_{i j, l}^{+} \delta x_{i} \delta x_{j}\right) \\
& +\frac{1}{2} \sum_{j=1}^{N^{\mathrm{s}}} k_{j, i}^{+} \delta x_{j} \delta x_{j}-\sum_{l=1}^{N^{\mathrm{s}}} k_{i i, l}^{+} \delta x_{i} \delta x_{i} .
\end{aligned}
$$

Because of the ordering of the $\circ$ product, this requires the elements of $\boldsymbol{L}^{\mathrm{ss,s}}$ to be defined as $\boldsymbol{L}_{j l, i}=k_{j l, i}^{+}-\left(\delta_{i j}+\delta_{i l}\right) \sum_{m=1}^{N^{s}} k_{j l, m}^{+}$for $j<l$ and $\boldsymbol{L}_{j \mathrm{j}, i}=\frac{1}{2} k_{j j, i}^{+}-\delta_{i j} \sum_{m=1}^{N^{s}} k_{i i, m}^{+}$. These coefficients encode the drift terms (2) for single subnetwork variables that depend on products of subnetwork variables.

The main structural features of (13) resemble the ones of the linear reduced dynamics. For instance, all subnetwork reactions are retained directly in the local-in-time terms, with rates appropriately arranged in $\boldsymbol{L}^{\mathrm{ss}}$ and $\boldsymbol{L}^{\mathrm{ss} s}$, while the bulk evolution appears via the additional nonlocal-in-time terms, divided by the projection approach into a memory contribution and a random force. The memory describes how the present state of a subnetwork species is affected by all past states of the subnetwork: the effect of single subnetwork species is modulated by a linear memory function $\boldsymbol{M}^{\text {ss }}\left(t-t^{\prime}\right)$ given by (11), whereas the effects of subnetwork products enter via a nonlinear memory function [see Eq. (4) in Sec. I of the supplementary material]; these memory functions have been analyzed in detail in Ref. 12.

Here, our focus will be on the structure of the random force $\boldsymbol{r}(t)$. Similar to the memory, we can write $\boldsymbol{r}(t)=\boldsymbol{r}_{0}(t)+\boldsymbol{r}_{1}(t)$ as the sum of a contribution from the linear dynamics $\boldsymbol{r}_{0}(t)$ and a nonlinear correction $\boldsymbol{r}_{1}(t)$. The former is given by

$$
\boldsymbol{r}_{0}^{\mathrm{T}}(t)=\delta \boldsymbol{x}^{\mathrm{b}^{\mathrm{T}}}(0) \boldsymbol{E}_{\mathrm{bb}}(t) \boldsymbol{L}^{\mathrm{bs}}
$$

Here, we have introduced the shorthand $\boldsymbol{E}(t)=e^{\mathbf{Q} \boldsymbol{Q} \boldsymbol{t}}$, where $\boldsymbol{Q}$ is the matrix form of the projector onto the bulk, so $\boldsymbol{E}_{\mathrm{bb}}(t)=e^{\mathrm{L}^{\mathrm{bb}}(t)}$ (see Sec. I of the supplementary material). $\boldsymbol{r}_{0}(t)$ is simply the random force featuring in the reduced linearized dynamics, so it corresponds to (12) in the limit $\varepsilon \rightarrow 0$. This limit represents the fact that the projection approach describes observables conditionally averaged over the intrinsic noise, leaving for the random force only the stochastic effects due to fluctuations in the bulk initial conditions.

The nonlinear random force $\boldsymbol{r}_{1}(t)$ cannot be calculated in closed form but like the matrix representation of the relevant operators can be calculated in an expansion in $\delta x$. This gives

$$
\begin{aligned}
\boldsymbol{r}_{1}^{\mathrm{T}}(t)= & \left(\delta \boldsymbol{x}^{\mathrm{s}}(0) \circ \delta \boldsymbol{x}^{\mathrm{b}}(0)\right)^{\mathrm{T}}\left[\boldsymbol{E}_{\mathrm{sb}, \mathrm{b}}(t) \boldsymbol{L}^{\mathrm{bs}}+\boldsymbol{E}_{\mathrm{sb}, \mathrm{sb}}(t) \boldsymbol{L}^{\mathrm{sb}, \mathrm{s}}\right] \\
& +\left(\delta \boldsymbol{x}^{\mathrm{b}}(0) \circ \delta \boldsymbol{x}^{\mathrm{b}}(0)\right)^{\mathrm{T}}\left[\boldsymbol{E}_{\mathrm{bb}, \mathrm{b}}(t) \boldsymbol{L}^{\mathrm{bs}}+\boldsymbol{E}_{\mathrm{bb}, \mathrm{sb}}(t) \boldsymbol{L}^{\mathrm{sb}, \mathrm{s}}\right]
\end{aligned}
$$

if we include terms up to $O\left(\delta x^{\mathrm{b}}(0)^{2}\right)$; higher order terms may reasonably be expected to be negligible in many (but not all) applications to reaction networks. $\boldsymbol{L}^{\mathrm{sb}, \mathrm{s}}$ is defined similar to $\boldsymbol{L}^{\mathrm{ss}, \mathrm{s}}$, i.e., contains the coefficients of products such as $\delta \boldsymbol{x}^{\mathrm{s}} \circ \delta \boldsymbol{x}^{\mathrm{b}}$, which are considered part of the bulk subspace, in the time evolution equations for linear subnetwork observables. Equations (15) and (16) will be the basis for all analysis of extrinsic noise properties below, as predicted by the projection approach. From them, one can also see that random force terms are present only for nonzero entries of the matrix blocks $\boldsymbol{L}^{\mathrm{bs}}$ and $\boldsymbol{L}^{\mathrm{sb}, \mathrm{s}}$, which contain the rates of the subnetwork biochemical reactions with bulk species. In other words, the only subnetwork concentrations whose evolution is affected by a random force (and a memory term) as in (13) are the ones directly reacting with bulk species: we will call such subnetwork species "boundary species."

\section{Colored noise in 3GA}

We next set out how extrinsic noise is obtained within our 3GA approach. ${ }^{13}$ The underlying path integral method follows the classical Martin-Siggia-Rose-Jansen-DeDominicis ${ }^{25-29}$ formulation for stochastic processes. It allows one to express stochastic dynamics, in our case of the biochemical concentrations in (1), as an integral over all possible time evolutions ("paths" or "trajectories") of the system. Reducing the dynamical description to the subnetwork in this representation becomes equivalent to marginalizing over the bulk trajectories. This task would be extremely difficult in general but becomes feasible, both conceptually and technically, when the joint probability distribution over subnetwork and bulk trajectories are Gaussian (as the marginal of a joint Gaussian is again Gaussian). 
Thus, the main idea of the $3 \mathrm{GA}^{13}$ is to start from a Gaussian approximation for this probability distribution, which leads to a linearization of Eq. (1) around the (in principle) time-dependent means. This Gaussian approximation is then used as a tractable reference distribution for obtaining corrections from nonlinear terms by perturbation theory, leading to a nonlinear subnetwork-reduced dynamics analogous to (13). (The method was referred to as Gaussian variational approximation in Ref. 13 where we used a variational approach to derive the baseline approximation.) To simplify the final expressions, we choose to linearize about the deterministic steady states, as we did in Sec. II B.

Here, we extend the analysis in Ref. 13 to include the intrinsic, multiplicative white noise $\boldsymbol{\xi}(t)$ with covariance (3) (see Sec. II of the supplementary material for the full derivation). We thus retain all stochastic terms, including the $\boldsymbol{x}$-dependencies of $\boldsymbol{\Sigma}(\boldsymbol{x})$, in the perturbative expansion. Substituting $\boldsymbol{x}(t)$ by $\boldsymbol{y}+\delta \boldsymbol{x}(t)$, we decompose (3) as

$$
\begin{aligned}
\boldsymbol{\Sigma}(\boldsymbol{x}(t))= & \varepsilon \boldsymbol{S} \operatorname{diag}(\boldsymbol{f}(\boldsymbol{x})) \boldsymbol{S}^{\mathrm{T}}=\varepsilon \boldsymbol{S}\left[\operatorname{diag}\left(\boldsymbol{f}_{0}\right)+\operatorname{diag}\left(\boldsymbol{f}_{1}(\delta \boldsymbol{x})\right)\right. \\
& \left.+\operatorname{diag}\left(f_{2}\left(\delta \boldsymbol{x}^{2}\right)\right)\right] \boldsymbol{S}^{\mathrm{T}}=\boldsymbol{\Sigma}_{0}+\boldsymbol{\Sigma}_{1}+\boldsymbol{\Sigma}_{2},
\end{aligned}
$$

where $f_{0}, f_{1}(\delta x)$, and $f_{2}\left(\delta x^{2}\right)$ denote, respectively, the reaction fluxes for $\delta x=0$ and the $\delta x$-dependent and $\delta x^{2}$-dependent pieces. The additive noise of the linearized dynamics corresponds to the first term, i.e., $\varepsilon \boldsymbol{S} \operatorname{diag}\left(\boldsymbol{f}_{0}\right) \boldsymbol{S}^{\mathrm{T}}=\boldsymbol{\Sigma}_{0}$; similarly, we identify $\varepsilon S \operatorname{diag}\left(f_{1}(\delta x)\right) S^{\mathrm{T}} \equiv \boldsymbol{\Sigma}_{1}$ and $\varepsilon S \operatorname{diag}\left(\boldsymbol{f}_{2}\left(\delta x^{2}\right)\right) \boldsymbol{S}^{\mathrm{T}} \equiv \boldsymbol{\Sigma}_{2}$. When we develop the perturbative expansion, we focus on first order corrections to the linear dynamics and so discard the last term $\boldsymbol{\Sigma}_{2}$ [with $O\left(\delta x^{2}\right)$ terms] that would contribute only at a higher order. The resulting nonlinear 3GA-reduced dynamical equations are

$$
\begin{aligned}
\frac{d}{d t} \delta \boldsymbol{x}^{\mathrm{s}^{\mathrm{T}}}(t)= & \delta \boldsymbol{x}^{\mathrm{s}^{\mathrm{T}}}(t) \boldsymbol{L}^{\mathrm{ss}}+\left(\delta \boldsymbol{x}^{\mathrm{s}}(t) \circ \delta \boldsymbol{x}^{\mathrm{s}}(t)\right)^{\mathrm{T}} \boldsymbol{L}^{\mathrm{ss}, \mathrm{s}} \\
& +\int_{0}^{t} d t^{\prime} \delta \boldsymbol{x}^{\mathrm{s}^{\mathrm{T}}}\left(t^{\prime}\right) \boldsymbol{M}^{\mathrm{ss}}\left(t-t^{\prime}\right) \\
& +\int_{0}^{t} d t^{\prime} \int_{0}^{t^{\prime}} d t^{\prime \prime}\left(\delta \boldsymbol{x}^{\mathrm{s}}\left(t^{\prime}\right) \circ \delta \boldsymbol{x}^{\mathrm{s}}\left(t^{\prime \prime}\right)\right)^{\mathrm{T}} \\
& \times \boldsymbol{M}_{3 \mathrm{GA}}^{\mathrm{ss} \mathrm{s}}\left(t, t^{\prime}, t^{\prime \prime}\right)+\chi^{\mathrm{T}}(t),
\end{aligned}
$$

where the times in $\boldsymbol{M}_{3 \mathrm{GA}}^{\mathrm{ss}, \mathrm{s}}\left(t, t^{\prime}, t^{\prime \prime}\right)$ are ordered as $t>t^{\prime}>t^{\prime \prime}$. We have used a subscript to distinguish $\boldsymbol{M}_{3 \mathrm{GA}}^{\mathrm{ss} s}\left(t, t^{\prime}, t^{\prime \prime}\right)$ explicitly from the nonlinear memory function of the projection approach $\boldsymbol{M}_{\mathrm{P}}^{\mathrm{ss}, \mathrm{s}}\left(t-t^{\prime}\right)$, which is rather different as clear already from its dependence on only a single time difference.

Note that the reduced dynamics (18) is valid for any noise level $\varepsilon$, i.e., all the intrinsic noise terms are still present in contrast to the projected equation (13) that relates to conditional averages over the intrinsic fluctuations. The overall effective noise in the $3 \mathrm{GA}$ on the subnetwork is given by $\chi(t)$ in (18). Similar to the random force, we can split this as $\chi(t)=\chi_{0}(t)+\chi_{1}(t)$, where $\chi_{0}(t)$ is the noise of the linearized dynamics (12), while $\chi_{1}(t)$ stems from the nonlinear corrections; hence, it contains $\delta x^{s}$-dependent terms as follows:

$$
\begin{aligned}
\boldsymbol{\chi}_{1}^{\mathrm{T}}(t)= & \boldsymbol{\xi}_{1}^{\mathrm{s}^{\mathrm{T}}}(t)+\int_{0}^{t} d t^{\prime \prime} \boldsymbol{\xi}_{1}^{\mathrm{b}^{\mathrm{T}}}\left(t^{\prime \prime}\right) \boldsymbol{E}_{\mathrm{bb}}\left(t-t^{\prime \prime}\right) \boldsymbol{L}^{\mathrm{bs}} \\
& +\left(\delta \boldsymbol{x}^{\mathrm{s}}(t) \circ \lambda(t)\right)^{\mathrm{T}} \boldsymbol{L}^{\mathrm{sb}, \mathrm{s}}+\int_{0}^{t} d t^{\prime \prime}\left[\left(\delta \boldsymbol{x}^{\mathrm{s}}\left(t^{\prime \prime}\right) \circ \lambda\left(t^{\prime \prime}\right)\right)^{\mathrm{T}} \boldsymbol{L}^{\mathrm{sb}, \mathrm{b}}\right. \\
& \left.+\left(\lambda\left(t^{\prime \prime}\right) \circ \lambda\left(t^{\prime \prime}\right)\right)^{\mathrm{T}} \boldsymbol{L}^{\mathrm{bb}, \mathrm{b}}\right] \boldsymbol{E}_{\mathrm{bb}}\left(t-t^{\prime \prime}\right) \boldsymbol{L}^{\mathrm{bs}}+\int_{0}^{t} d t^{\prime} \int_{0}^{t^{\prime}} d t^{\prime \prime} \\
& \times\left[\left(\lambda\left(t^{\prime}\right) \circ \boldsymbol{E}_{\mathrm{bb}}^{\mathrm{T}}\left(t^{\prime}-t^{\prime \prime}\right)\left(\boldsymbol{L}^{\mathrm{sb}}\right)^{\mathrm{T}} \delta \boldsymbol{x}^{\mathrm{s}}\left(t^{\prime \prime}\right)+\boldsymbol{E}_{\mathrm{bb}}^{\mathrm{T}}\left(t^{\prime}-t^{\prime \prime}\right)\right.\right. \\
& \left.\left.\times\left(\boldsymbol{L}^{\mathrm{sb}}\right)^{\mathrm{T}} \delta \boldsymbol{x}^{\mathrm{s}}\left(t^{\prime \prime}\right) \circ \lambda\left(t^{\prime}\right)\right)^{\mathrm{T}} \boldsymbol{L}^{\mathrm{bb}, \mathrm{b}}\right] \boldsymbol{E}_{\mathrm{bb}}\left(t-t^{\prime}\right) \boldsymbol{L}^{\mathrm{bs}} .
\end{aligned}
$$

There is some notation to explain here. As in Sec. II C, the matrices $\boldsymbol{L}^{\mathrm{sb}, \mathrm{s}}, \boldsymbol{L}^{\mathrm{sb}, \mathrm{b}}$, and $\boldsymbol{L}^{\mathrm{bb}, \mathrm{b}}$ contain the sets of rate constants involved, respectively, in the reaction of a bulk and subnetwork species to give a complex in the subnetwork $\left(\boldsymbol{L}^{\mathrm{sb}, \mathrm{s}}\right)$ or in the bulk $\left(\boldsymbol{L}^{\mathrm{sb}, \mathrm{b}}\right)$, or in two bulk species $\left(\boldsymbol{L}^{\mathrm{bb}, \mathrm{b}}\right)$ forming another bulk species. For brevity, and in line with our assumptions on subnetwork modeling set out in Sec. II A, we have already set $\boldsymbol{L}^{\mathrm{bb}, \mathrm{s}}=\boldsymbol{L}^{\mathrm{ss}, \mathrm{b}} \equiv 0$, i.e., we assume there are no subnetwork complexes formed from two bulk species or conversely bulk complexes from two subnetwork species; the same convention was used tacitly already in (16). The relevant matrix blocks can easily be re-instated if desired (see, for example, Ref. 13 and Sec. II of the supplementary material). A subscript 0 on $\boldsymbol{\xi}$ labels a noise component whose covariance is independent of $\delta \boldsymbol{x}$, while $\xi_{1}$ carry the remaining $\delta \boldsymbol{x}$-dependent parts,

$$
\left\langle\xi_{0}(t) \xi_{0}^{\mathrm{T}}\left(t^{\prime}\right)\right\rangle=\Sigma_{0}^{*} \delta\left(t-t^{\prime}\right), \quad \xi_{1}(t)=\frac{1}{2}\left(\Sigma_{1} \Sigma_{0}^{-1} \xi_{0}\right)^{\cdot}(t) \cdot=\mathrm{s}, \mathrm{b} .
$$

Expression (20) for $\xi_{1}$ is obtained from an expansion of $\sqrt{\boldsymbol{\Sigma}}$ in $\delta \boldsymbol{x}$ up to first order; it thus preserves the $\sim \delta \boldsymbol{x}$ dependence of the noise covariance expected as a leading correction to a Gaussian solution (see Sec. II of the supplementary material). Note that $\xi_{1}(t)$, being linearly related to $\xi_{0}(t)$, does not add stochasticity per se but simply corrects the correlation structure by accounting (multiplicatively) for the $\delta \boldsymbol{x}$-dependence. In (19), we have further defined the shorthand

$$
\lambda^{\mathrm{T}}(t)=\delta \boldsymbol{x}^{\mathrm{b}^{\mathrm{T}}}(0) \boldsymbol{E}_{\mathrm{bb}}(t)+\int_{0}^{t} d t^{\prime} \boldsymbol{\xi}_{0}^{\mathrm{b}^{\mathrm{T}}}\left(t^{\prime}\right) \boldsymbol{E}_{\mathrm{bb}}\left(t-t^{\prime}\right),
$$

whose covariance $\left\langle\lambda(t) \lambda^{\mathrm{T}}\left(t^{\prime}\right)\right\rangle=C^{\mathrm{bb} \mid \mathrm{s}}\left(t, t^{\prime}\right)$ has the meaning of a bulk-bulk covariance matrix conditioned on knowing the subnetwork trajectory

$$
\begin{aligned}
\boldsymbol{C}^{\mathrm{bb} \mid \mathrm{s}}\left(t, t^{\prime}\right)= & \boldsymbol{E}_{\mathrm{bb}}^{\mathrm{T}}(t) \boldsymbol{C}^{\mathrm{bb}}(0,0) \boldsymbol{E}_{\mathrm{bb}}\left(t^{\prime}\right) \\
& +\int_{0}^{\min \left(t, t^{\prime}\right)} d t^{\prime \prime} \boldsymbol{E}_{\mathrm{bb}}^{\mathrm{T}}\left(t-t^{\prime \prime}\right) \boldsymbol{\Sigma}_{0}^{\mathrm{bb}} \boldsymbol{E}_{\mathrm{bb}}\left(t^{\prime}-t^{\prime \prime}\right) .
\end{aligned}
$$

Like for the nonlinear memory, the nonlinear effective noise term in our marginalization approach does not coincide with its ana$\log$ in the projection method, the random force; this is true even in the limit $\varepsilon \rightarrow 0$. In particular, (19) contains a dependence on the whole trajectory $\delta x^{\mathrm{s}}\left(t^{\prime}\right)$. Nonetheless, in the limit $\varepsilon \rightarrow 0$ of vanishing intrinsic noise, the combination of memory and effective noise still gives an approximation equivalent to the combination of memory and random force in the projection method, up to $O\left(\delta x^{s 2}\right)$. For $\varepsilon>0$, the two approximations become different as the random force [(15) and (16)] contains only the extrinsic noise from the uncertainty about the bulk initial state, while the 
effective colored noise [(12) and (19)] contains also the effects of intrinsic fluctuations, i.e., the subnetwork intrinsic noise $\left(\xi_{0}^{s}\right.$ and $\left.\xi_{1}^{s}\right)$ and bulk intrinsic noise $\left(\xi_{0}^{\mathrm{b}}\right.$ and $\left.\xi_{1}^{\mathrm{b}}\right)$. The 3GA-reduced equations thus provide a more complete picture of fluctuations in the subnetwork dynamics: they retain the original subnetwork intrinsic randomness as well as extrinsic (bulk-related) components, i.e., the unknown bulk initial conditions and the propagation across the network of bulk intrinsic noise. In Secs. III and IV below, we test the accuracy of the subnetwork equations, depending on which of these contributions to the extrinsic noise is retained in the evaluation.

\section{ACCURACY OF SUBNETWORK EQUATIONS WITH INITIAL UNCERTAINTY IN THE BULK}

In the following, we assess how well our explicit expressions for extrinsic noise capture the two contributions identified above: (i) initial deviations of the bulk from the steady state and (ii) intrinsic stochastic fluctuations in the bulk dynamics, which are captured only by the $3 \mathrm{GA}$. In this section, we address contribution (i). Without intrinsic noise $(\varepsilon \rightarrow 0)$, the projection and 3GA methods give results at a nonlinear level that agree up to $O\left(\delta x^{\text {s2 }}\right)$ but not beyond and only when the combination of memory and effective noise is taken into account; ${ }^{13}$ thus, a comparison between the two methods will also be of interest.

\section{A. Measures of accuracy}

We sample bulk initial conditions from Gaussian distributions centered around zero with variance equal to $\left\langle\delta x_{i}^{2}\right\rangle=\varepsilon_{0} y_{i}$ for $i=1, \ldots, N^{\mathrm{b}}$, introducing a parameter $\varepsilon_{0}$ separate from $\varepsilon$ that sets the amplitude of initial fluctuations. The scaling with $y_{i}$ corresponds to the Gaussian (large copy number) limit of Poisson fluctuations in the number of molecules from each individual bulk species. The parameter $\varepsilon_{0}$ is the inverse of an effective volume that converts steady state concentrations $y_{i}$ to copy numbers $y_{i} / \varepsilon_{0}$.

We fix the subnetwork initial conditions, randomly sample bulk initial conditions, and then solve both the full reaction equations and the reduced subnetwork equations. Repeating this process, we get at each point in time a distribution of predicted subnetwork concentrations, one according to each of the reduced equations (projection or $3 \mathrm{GA}$ ) and one according to the full nonlinear reaction equations (here the quantity $\delta x$ is simply found by the change of variable $\delta x=x-y$ from the full nonlinear solution $x$ ). Our goal is to assess how close these two distributions are, i.e., to quantify how well the reduced equations capture fluctuations due to variation in bulk initial conditions. One possible measure of similarity between the true distribution $p(\delta x)$ and the approximated one $q(\delta x)$ is the Kullback-Leibler (KL) divergence. ${ }^{30}$ If we focus on the distributions of a single concentration variable and approximate these as Gaussians $p(\delta x)=N\left(\mu, \sigma^{2}\right)$ and $q(\delta x)=N\left(\hat{\mu}, \hat{\sigma}^{2}\right)$, then

$$
\mathrm{KL}(p \| q)=\frac{1}{2} \ln \frac{\hat{\sigma}^{2}}{\sigma^{2}}+\frac{\sigma^{2}-\hat{\sigma}^{2}+(\hat{\mu}-\mu)^{2}}{2 \hat{\sigma}^{2}} .
$$

The Gaussian approximation is exact for the linearized dynamics and proved to be a good approximation in our numerical experiments for the solution of the full nonlinear CLE [see Fig. S1(a)].
As a consequence, the Gaussian KL estimates are consistent with a non-parametric estimate [see Fig. S1(b)] that makes no assumption on the form of the distributions. As Fig. S1(b) demonstrates, using Gaussian fits for the KL divergence has the advantage of giving substantially less noisy estimates; it is also computationally faster. We note that large KLs [above $O(1)$ ] indicate that the distributions being compared are quite different, with the integrand in $\operatorname{KL}(p \| q)=\int \delta x$ $p(\delta x) \ln [p(\delta x) / q(\delta x)]$ picking up contributions in the tails of $q(\delta x)$ where a Gaussian fit may be insufficient. Our KL estimates in this regime will therefore not be quantitatively accurate, and we will only use them for qualitative comparisons, e.g., between KL values of different orders of magnitude. We note finally that alternative definitions of distance between steady state distributions in stochastic models of biochemical reaction networks have been proposed, such as the Wasserstein distance. ${ }^{31}$ We have verified that, when evaluating the Wasserstein distance, the ordering in accuracy among the different approximations we consider and the trends in time are the same as for our Gaussian KL divergence estimates [see Fig. S1(c)]. We therefore report results only for the latter.

Returning then to (23), to evaluate the Gaussian KL, we just need to collect the relevant single-species means and variances. Such a single-species KL is useful to measure how accurately means and variances are captured for individual species, e.g., those immediately affected by the interaction with the bulk, which we will call boundary species. At the numerical level, we will work with dimensionless concentrations, namely, fractional deviations from the steady state values $\delta \tilde{x}=\delta x / y$ (the definitions of $\mathrm{KL}$ are invariant under this change of variables).

An alternative measure that is sensitive to the accuracy of correlations between the fluctuations of different subnetwork concentrations at equal times is the normalized mean square error

$$
\Delta C=\frac{\sum_{i, j=1}^{N^{s}}\left(C_{i j}-\hat{C}_{i j}\right)^{2} /\left(y_{i} y_{j}\right)^{2}}{\sum_{i, j=1}^{N^{s}} C_{i j}^{2} /\left(y_{i} y_{j}\right)^{2}},
$$

where $C_{i j}$ are the elements of the subnetwork correlation matrix $C^{s s}(t, t)$ from the full reaction equations and $\hat{C}_{i j}$ are those of the correlation matrix estimated from a reduction method (3GA or projection). The error $\Delta C$ is constructed in such a way that $\Delta C \sim 0$ when $\hat{C}_{i j}$ approximates well $C_{i j}$ and $\Delta C \sim 1$ when $\hat{C}_{i j}$ significantly underestimates $C_{i j}$. The factors $\left(y_{i} y_{j}\right)^{2}$ indicate that we are again looking at fractional deviations from steady states.

In order to illustrate the general features of our approach, we will often use the small reaction network shown in Fig. 1.

\section{B. Quantitative tests on a simple network model}

We sampled 1000 bulk initial conditions at $\varepsilon_{0}=0.1$ and then calculated the single-variable KL divergence (23) between the distributions predicted by the full reaction equations and the reduced (projected or 3GA) equations. We focus here on the two subnetwork boundary species (see Fig. 1), which will have extrinsic noise acting on them due to the interaction with the bulk environment. We work with fixed subnetwork initial conditions $\delta \tilde{x}_{1}(0)=-1$, $\delta \tilde{x}_{2}(0)=2, \delta \tilde{x}_{12}(0)=1$, i.e., a subnetwork state where the initial deviations from steady state are substantial, ranging from $-100 \%$ to $+200 \%$ across the three subnetwork species. Solving the subnetwork equations with the full time-dependence of $\boldsymbol{r}(t)$ from (15) and 


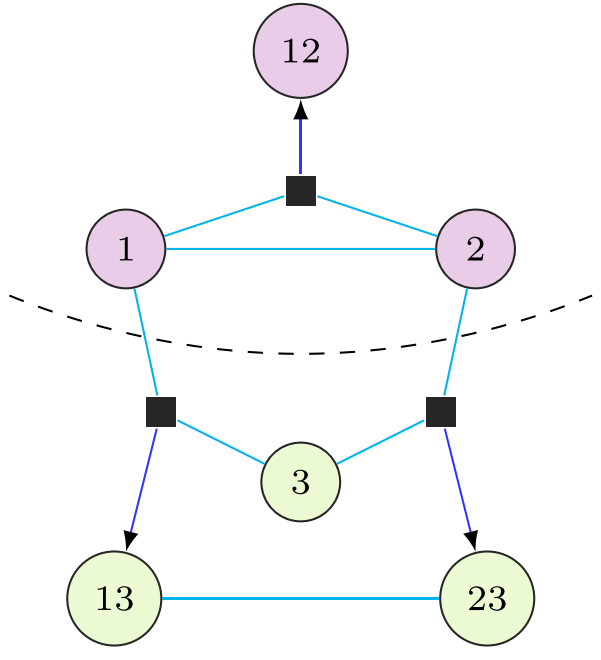

FIG. 1. Simple protein network. Sketch of a simple protein interaction network composed of six species (details specified in Appendix C). Species 1, 2, and 12 belong to the subnetwork (light red), and species 3,13 , and 23 form the bulk (light green). Squares and arrows indicate that protein 1 reacts with protein 2 to form complex 12 and in reverse 12 dissociates into 1 and 2 , with analogous reactions between 1 and 3 to form 13, and 2 and 3 to form 23 . Species 1 and 2 lie at the boundary of the subnetwork, i.e., they directly react with bulk species, while species 12 is internal to the subnetwork.

(16) and $\chi(t)$ from (12) and (19), we see that both model reduction approaches reproduce the exact dynamics extremely well at the level of single trajectories [Figs. 2(a)-2(c)] and of means and variances [Fig. 2(d)]. This is confirmed by the low values of the KL divergence
[Fig. 2(e)]. The small residual KL values show that the two reduction methods perform better in different time regimes (3GA at short times and projection method at long times). Note that even though the projection and 3GA reduction methods are known theoretically to be equivalent only to $O\left(\delta x^{2}\right)$, they agree rather well with each other and with the full dynamics even for the large initial subnetwork deviations from steady state explored in Fig. 2, suggesting that higher order corrections in $\delta \boldsymbol{x}$, while present, in principle, are quantitatively small. Similarly, although the nonlinear random force $\boldsymbol{r}(t)$ and nonlinear colored noise $\chi(t)$ are not defined in the same way, they both capture the extrinsic noise from initial bulk fluctuations and we observe that structurally they look very similar (Fig. 3). We note in this context that the means of $\boldsymbol{r}(t)$ and $\boldsymbol{\chi}(t)$, as evaluated by averaging over many realizations of bulk initial conditions, are different from zero. This is due to terms $\delta x^{\mathrm{b}}(0) \circ \delta x^{\mathrm{b}}(0)$ that are present both in $\boldsymbol{r}_{1}(t)(16)$ and $\boldsymbol{\chi}_{1}(t)$ [via $\boldsymbol{\lambda} \circ \boldsymbol{\lambda}$ in (19)]: when averaged, these terms scale as $C^{\mathrm{bb}}(0,0) \sim \mathcal{E}_{0}$ rather than vanishing exactly. The nonzero mean, arising from nonlinear propagation of stochastic effects, can be in principle subtracted and added back as a $t$-dependent piece in the subnetwork equations. However, this would be rather involved without providing any conceptual advantage.

\section{C. "Impulse plus persistent" extrinsic noise approximation}

In practice, it can be difficult to explicitly calculate $\boldsymbol{r}(t)$ or $\chi(t)$ as functions of time, and we therefore explore ways of approximating these extrinsic noise terms. Intuition can be gained from Fig. 3(a), which shows the random force $r^{1}$ and the colored noise $\chi^{1}$ acting on $\delta \tilde{x}_{1}$ for three different sets of bulk initial conditions sampled at $\mathcal{E}_{0}=0.1$. One sees that the extrinsic noise can have a constant piece

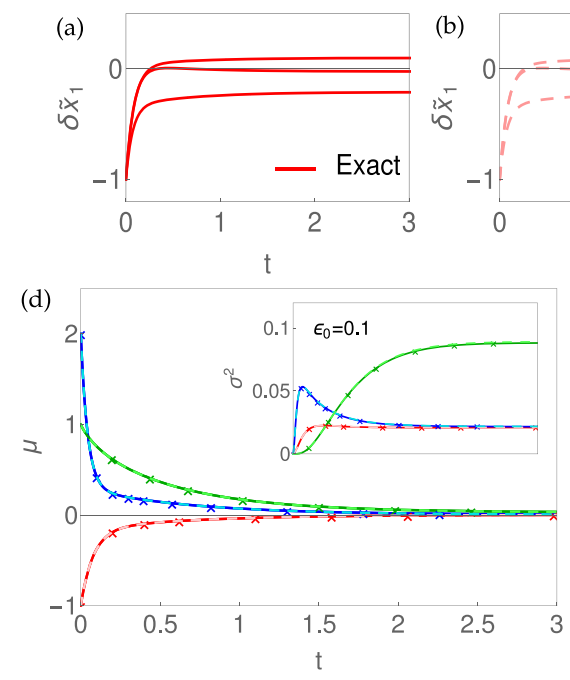

FIG. 2. Subnetwork dynamics with extrinsic noise in the simple network model. Time courses of the subnetwork boundary species $\delta \tilde{x}_{1}$ of the simple network model of Fig. 1: (a) exact, bold lines; (b) 3GA, dashed lines; and (c) projection, bold lines with crosses. In each plot, single trajectories correspond to 3 different realizations [the same in (a)-(c)] of random bulk initial conditions at $\varepsilon_{0}=0.1$ : their effect is visible both on the transient and the long time behavior. For each of them, the exact trajectory in (a) is accurately reproduced by $3 G A$ [corresponding curve in (b)] and by projection methods [corresponding curve in (c)]. (d) Exact subnetwork means over 1000 random bulk initial conditions compared to the approximated means by 3GA/projection methods [same line style as in (a)-(c)]. The inset shows the comparison of variances. (e) Subnetwork $\mathrm{KLs}$ for full $3 \mathrm{GA}$ and full projected equations. The difference in the long time behavior, induced by random bulk initial conditions, is less well captured by the $3 \mathrm{GA}$, where the errors on means and variance both reach nonzero values at long times, while the initial transient is less well captured by projection methods. 

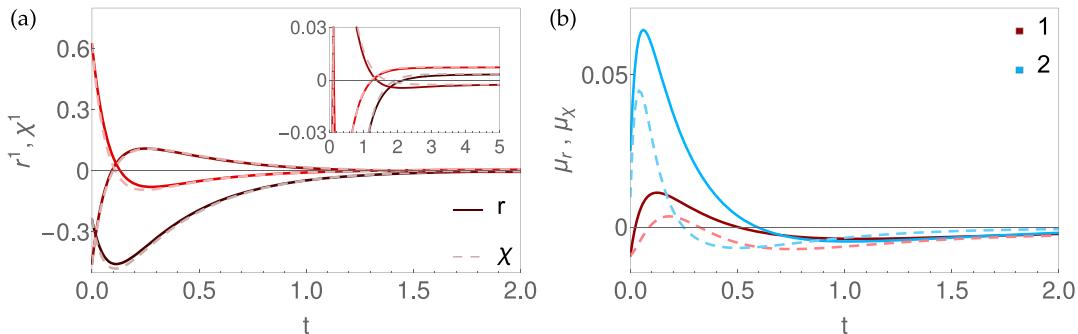

FIG. 3. Extrinsic noise from random bulk initial conditions at $\varepsilon_{0}=0.1$ in the simple network model. (a) Random force on $\delta \tilde{x}_{1}$ (solid lines) and $3 \mathrm{GA}$ colored noise (dashed lines) for a sample of three different initial conditions. The zoom in the inset shows that there is a small but nonzero asymptote on each curve. (b) Mean random force (over 1000 bulk initial conditions) on $\delta \tilde{x}_{1}$ and $\delta \tilde{x}_{2}$ (solid lines) and mean $3 G A$ colored noise (dashed lines).

for large times, as well as contribution that is significant only for short times. The long-time piece arises from the fact that different bulk initial conditions can produce different values of quantities (specifically weighted sums of certain molecule numbers) that are conserved in our example network.

A more systematic analysis, presented in detail in Appendix A, shows that in the projected subnetwork equations, one obtains the correct random force-dependent steady state from an approximate form of the random force as long as the approximation preserves both its long-time limit $\boldsymbol{r}_{\infty}$ and the total contribution from the short-time transient $\overline{\Delta r}=\int_{0}^{\infty} d t^{\prime}\left[\boldsymbol{r}\left(t^{\prime}\right)-\boldsymbol{r}_{\infty}\right]$. This suggests an approximation where the transient part of the random force is replaced by a single impulse at $t=0$, specifically

$$
\boldsymbol{r}(t) \simeq \boldsymbol{r}_{\infty}+\delta(t) \overline{\Delta \boldsymbol{r}}
$$

This can then be approximated further by only taking into account one of the two contributions above. By direct analogy, the extrinsic noise in the $3 \mathrm{GA}$ approach can be approximated as

$$
\chi(t) \simeq \chi_{\infty}+\delta(t) \overline{\Delta \chi},
$$

where $\chi_{\infty}=\lim _{t \rightarrow \infty} \chi(t)$ and $\overline{\Delta \chi}=\int_{0}^{\infty} d t^{\prime}\left[\chi\left(t^{\prime}\right)-\chi_{\infty}\right]$.

Our numerical tests of these approximations show that retaining only the persistent piece of $\boldsymbol{r}(t)$ and $\chi(t)$ is not a good approximation, resulting in very large KL divergences (data not shown). This makes sense given that in Fig. 3(a), one sees that the constant pieces of $\boldsymbol{r}(t)$ and $\boldsymbol{\chi}(t)$ can be small compared to the transients.

Once the transient piece of the extrinsic noise is included approximately as in (25) and (26), respectively, much more satisfactory predictions for the subnetwork dynamics are found. The KL divergences [Fig. 4(a)] are small and close to those found previously with the full form of the random force or effective noise, except at very short times-here, the fact that we are not fully capturing the time-dependence of the extrinsic noise transients evidently matters. The deviations $\Delta C$ in the predicted covariances show the same trend, being substantial only for short times and small [but still larger than when using the full form of $\boldsymbol{r}(t)$ or $\chi(t)$ ] at long times. In the example shown, one can in fact drop the persistent part of the extrinsic noise while still getting very accurate subnetwork dynamics predictions (Fig. 4), although we have seen in other tests that this is not generically true (see, e.g., Sec. IV B of the supplementary material). Note finally that the properties of the "impulse plus persistent" approximation for the extrinsic noise that we have seen so far are also observed in the simpler case of dynamics linearized around the fixed point (data not shown). The only difference is that the subnetwork equations with the full form of the extrinsic noise are exact here and so give zero KL divergences up to sampling errors.

\section{Variation with strength $\varepsilon_{0}$ of initial bulk fluctuations}

So far, we have analyzed how accurately the two model reduction methods can capture initial bulk fluctuations of fixed strength
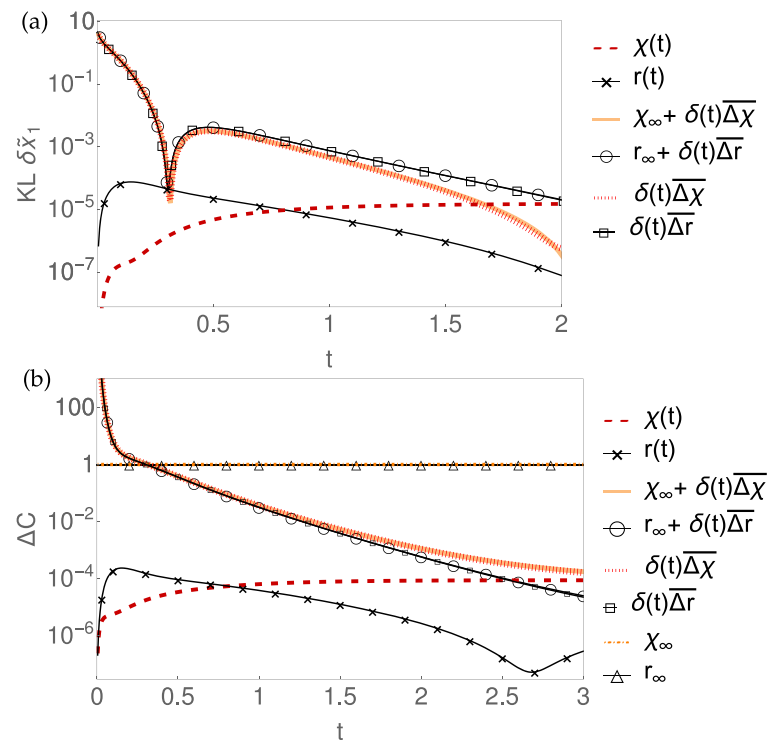

FIG. 4. Accuracy of extrinsic noise approximations at $\varepsilon_{0}=0.1$. (a) Comparison of $\mathrm{KLs}$ for $\delta \tilde{x}_{1}$ for projected equations (solid lines with symbols) and $3 \mathrm{GA}$ equations (dashed lines). Lowest two curves: full extrinsic noise; the other four curves show the approximation of the noise by an initial impulse and a persistent piece, and by only an initial impulse, as shown in the legend; the $\mathrm{KL}$ divergences remain small except in an initial transient. (b) Comparison of errors on $C, \Delta C$, for the 3GA and projected equations and for the same approximations of the random force $r(t)$ and the colored noise $\chi(t)$, respectively. The results are shown in addition for the approximation containing only the persistent piece: here, the value $\Delta C \sim 1$ indicates that the true covariances are strongly underestimated. 

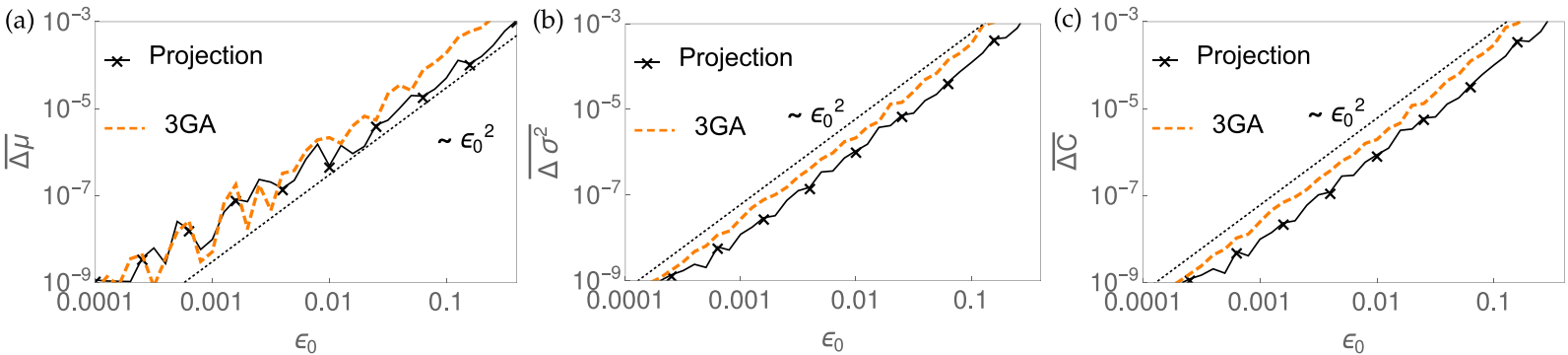

FIG. 5. Variation with $\varepsilon_{0}$ of integrated error on subnetwork means (a), variances (b), and correlations (c) over a sample of 1200 random bulk initial conditions for projected and $3 \mathrm{GA}$ equations. Initial conditions in the subnetwork are set to steady state, $\delta \mathbf{x}^{\mathrm{s}}(0)=0$ (see text for discussion)

$\varepsilon_{0}$. Now, we vary $\varepsilon_{0}$ to assess the impact of the size of these fluctuations. Here, it is useful to look at summary measures of accuracy over entire trajectories, so we consider the absolute error in the mean concentrations, averaged over the subnetwork species and over the chosen total time window $T$,

$$
\overline{\Delta \mu}=\frac{1}{T N^{s}} \int_{0}^{\mathrm{T}} d t \sum_{i=1}^{N^{\mathrm{s}}} \frac{1}{y_{i}}\left|\mu_{i}(t)-\hat{\mu}_{i}(t)\right| .
$$

The normalization by $y_{i}$ ensures that this quantity is dimensionless, again measuring errors in fractional deviations from steady state values. Similarly, we consider the errors in the variances

$$
\overline{\Delta \sigma^{2}}=\frac{1}{T N^{s}} \int_{0}^{\mathrm{T}} d t \sum_{i=1}^{N^{\mathrm{s}}} \frac{1}{y_{i}^{2}}\left|\sigma_{i}^{2}(t)-\hat{\sigma}_{i}^{2}(t)\right|
$$

and in the full covariance matrix, which includes correlations between species

$$
\overline{\Delta C}=\frac{1}{T\left(N^{s}\right)^{2}} \int_{0}^{\mathrm{T}} d t \sum_{i, j=1}^{N^{s}} \frac{1}{y_{i} y_{j}}\left|C_{i j}(t)-\hat{C}_{i j}(t)\right| .
$$

Here, different from definition (24), we do not take square of the errors $\left|C_{i j}(t)-\hat{C}_{i j}(t)\right|$ and we do not normalize them by the exact correlations in order to avoid having a potentially $\varepsilon_{0}$-dependent normalization constant. To get an understanding of the expected scalings of $\overline{\Delta \mu}, \overline{\Delta \sigma^{2}}$, and $\overline{\Delta C}$ with $\varepsilon_{0}$, we assume the subnetwork initial conditions to be at steady state, $\delta x^{\mathrm{s}}(0)=0$. Other initial conditions would generate small errors, of order $\delta x^{s}(0)^{3}$ in $\overline{\Delta \mu}$, even for $\varepsilon_{0}$ : these arise from the truncation to quadratic terms in our projection (see Ref. 12 and Sec. I of the supplementary material). Subnetwork steady state initial conditions therefore allow us to isolate the effects of $\varepsilon_{0}$. (For general initial conditions, one broadly expects that the truncation error or the one from $\varepsilon_{0}$ will dominate, depending on which is larger.)

The desired error scaling with $\varepsilon_{0}$ can now be inferred from the construction of the projection and 3GA methods, which both involve neglecting terms beyond a certain order in the initial bulk fluctuations. As we are still considering $\varepsilon \rightarrow 0$ (no intrinsic noise), variances and covariances of subnetwork quantities are only driven by initial bulk fluctuations and must scale with $\varepsilon_{0}$ to leading order. As explained in Appendix B, the next terms of $O\left(\varepsilon_{0}^{2}\right)$ are already not treated fully systematically by projection or $3 \mathrm{GA}$, so the errors in means, variances, and covariances, $\overline{\Delta \mu}, \overline{\Delta \sigma^{2}}$, and $\overline{\Delta C}$, are expected to scale as $\varepsilon_{0}^{2}$.

These scalings are confirmed numerically in Fig. 5 over a wide range of $\varepsilon_{0}$, with deviations appearing only for very small $\varepsilon_{0}$ where the errors become too small to estimate reliably. Note that the errors on both means and (co-)variances remain low (below $10^{-3}$ ) even at the largest $\varepsilon_{0}=0.4$ we used, which corresponds to very substantial initial bulk fluctuations [because steady state copy numbers $y_{i} / \varepsilon_{0}$ in our model are then as small as $O(10)]$.

\section{ACCURACY OF SUBNETWORK EQUATIONS WITH INTRINSIC FLUCTUATIONS}

So far, we have restricted ourselves to the limit of noiseless dynamics, i.e., $\varepsilon \rightarrow 0$. As a next step, we consider the intrinsic (white Gaussian) noise $\boldsymbol{\xi}(t)$ from (1) for all subnetwork and bulk species while setting $\varepsilon_{0}=0$. Examples of single trajectories for different realizations of the noise are shown in Figs. 6(a)-6(c) for the simple protein interaction network of Fig. 1.

As projection methods provide only trajectories conditionally averaged over the noise, we need to use the 3GA to analyze the nonlinear dynamics in the presence of such fluctuations. ${ }^{32}$ We will compare its performance to the Steady State Linear Noise Approximation (ssLNA), ${ }^{16,20}$ which is based on the assumption that the bulk dynamics are fast enough to reach equilibrium with respect to the instantaneous subnetwork state, and to intermediate approximations that retain only some features of the effective noise $\chi(t)$ on the subnetwork dynamics, as summarized schematically in Table I. Building such intermediate approximations allows us to assess the impact of the different extrinsic noise contributions that arise in the process of model reduction, such as nonlinearity and time correlations.

The first intermediate approximation we explore is "linear noise," where in the 3GA-reduced dynamics (18), we keep the nonlinear memory [i.e., the integrals containing $\boldsymbol{M}^{\mathrm{ss}}\left(t-t^{\prime}\right)$ and $\left.\boldsymbol{M}_{3 \mathrm{GA}}^{\mathrm{ss} s}\left(t, t^{\prime}, t^{\prime \prime}\right)\right]$ but we replace the nonlinear (hence multiplicative) and colored effective noise $\chi(t)=\chi_{0}(t)+\chi_{1}(t)$ with its linear (hence additive), but still time-correlated, part $\chi_{0}(t)$ from (12). We refer to this approximation as $3 \mathrm{GA}-\boldsymbol{\chi}_{0}$.

We further study a "white linear noise" version of the $3 \mathrm{GA}-\chi_{0}$ where, in analogy to the ssLNA, we assume that the bulk dynamics is fast with respect to the subnetwork dynamics so that we can 


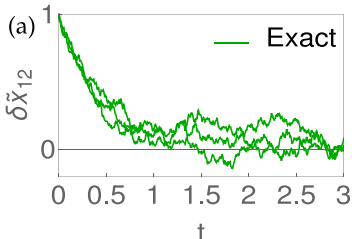

(d)

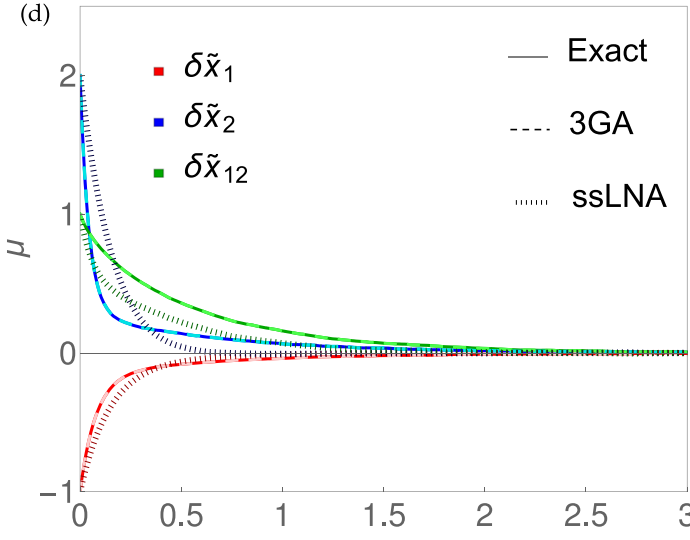

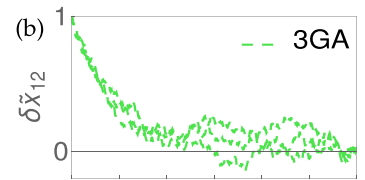

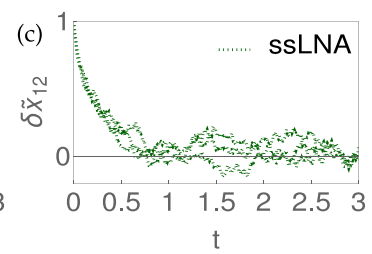

(e)

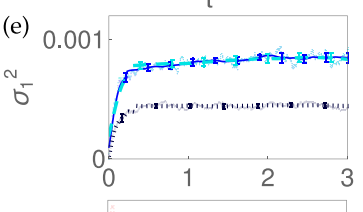

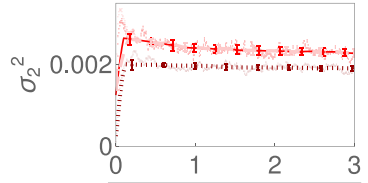

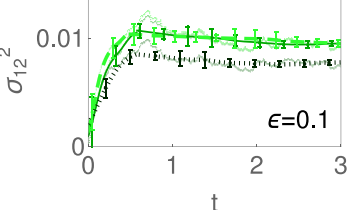

FIG. 6. Subnetwork dynamics with intrinsic noise in the simple network model. Examples of stochastic trajectories for subnetwork species $\delta \tilde{x}_{12}$ : (a) exact dynamics (bold curves), (b) 3GA model reduction (dashed curves), and (c) ssLNA (dotted curves). The different trajectories in each plot correspond to three different realizations of white noise with $\varepsilon=0.1$, and the same three noise realizations were used in each case to emphasize the differences arising from the distinct nature of the approximations. The bulk is initially taken at steady state, i.e., $\varepsilon_{0}=0$. Each exact trajectory in (a) is accurately reproduced by the $3 \mathrm{GA}$ [corresponding curve in (b)] but less well by ssLNA [corresponding curve in (c)]. Next, we compare exact means (d) and variances (e), calculated from a sample of 1000 noise realizations, to the ones given by the $3 G A$ and ssLNA [same line styles as in (a)-(c)]. The numerical results for the variances [points in (e)] have been smoothed in time for better visibility, with a representative set of error bars shown that have been estimated from the smoothing bins.

ignore the time correlations it induces in the effective noise. We thus approximate $\chi_{0}(t)$ by a white (uncorrelated in time) noise $\chi_{0}^{w}(t)$ with a time-dependent covariance matrix. The latter is determined such that the covariance of the cumulative noise $\chi_{0}^{w}(t)$ over a short time interval $t \ldots t+d t$ is the same as for the linear effective noise $\chi_{0}(t)$; here, the time interval $d t$ should be short compared to the dynamics of the subnetwork but larger than the timescale on which bulk fluctuations are correlated. Under these assumptions, one finds explicitly

$$
\chi_{0}^{w \mathrm{~T}}(t)=\boldsymbol{\xi}_{0}^{\mathrm{s}^{\mathrm{T}}}(t)-\boldsymbol{\xi}_{0}^{\mathrm{b}^{\mathrm{T}}}(t) \boldsymbol{L}^{\mathrm{bb}-1} \boldsymbol{L}^{\mathrm{bs}}
$$

The resulting approximation is Markovian at the level of the stochastic part but stays non-Markovian in its deterministic part as it retains the full nonlinear memory of the $3 \mathrm{GA}$; we will refer to it as $3 \mathrm{GA}-\chi_{0}^{w}$. As an aside, we note that $\chi_{0}^{w}(t)$ is identical to the ssLNA effective noise evaluated at the subnetwork steady state rather than the transient subnetwork concentrations used in the ssLNA (see Refs. 16 and 20).

\section{A. Quantitative tests on a simple network model}

We can assess the accuracy of the 3GA compared to the ssLNA and the intermediate approximations introduced above in terms of

TABLE I. Comparison of features of projection methods, 3GA, and the different approximate model reduction approaches to dynamics with intrinsic noise discussed in Sec. IV listed in descending order of sophistication. "n.s." is shorthand for "noise source."

\begin{tabular}{|c|c|c|c|c|}
\hline Approximation & Effective noise: from $\Sigma$ & From $C^{\mathrm{bb}}(0,0)$ & Memory & Terms \\
\hline Projection & $x$ & $\checkmark$ & $\checkmark$ & Eq. (13) with (15) and (16) \\
\hline $3 \mathrm{GA}$ & Nonlinear, multiplicative, colored & $\checkmark$ & $\checkmark$ & Eq. (18) with (12) and (19) \\
\hline 3GA, additive n.s. & Nonlinear, additive, colored & $\checkmark$ & $\checkmark$ & Eq. (18) with (12) and (19); $\xi_{1}$ dropped from (19) \\
\hline $3 \mathrm{GA}-\chi_{0}$, multiplicative n.s. & Linear, multiplicative, colored & $\checkmark$ & $\checkmark$ & Eq. (18) with (12); $\boldsymbol{\xi}_{0} \rightarrow \boldsymbol{\xi}_{0}+\boldsymbol{\xi}_{1}\left[\boldsymbol{\xi}_{1}\right.$ from Eq. (20)] \\
\hline $3 \mathrm{GA}-\chi_{0}$ & Linear, additive, colored & $\checkmark$ & $\checkmark$ & Eq. (18) with (12) \\
\hline $3 \mathrm{GA}-\boldsymbol{\chi}_{0}^{w}$ & Linear, additive, white & $\checkmark$ & $\checkmark$ & Eq. (18) with (12) approximated as white \\
\hline ssLNA & Linear, multiplicative, white & $x$ & $x$ & See Refs. 16 and 20 \\
\hline
\end{tabular}


the KL divergence (23) and the covariance accuracy $\Delta C$ (24). To estimate the required statistics, we use a sample of 1000 realizations of intrinsic noise with $\varepsilon=0.1$ (we set $\varepsilon_{0}=0$ to focus on the $\varepsilon$ dependence). When comparing the four approximations discussed so far, the difference between $3 \mathrm{GA}$ and $3 \mathrm{GA}-\chi_{0}$ quantifies the importance of nonlinearity of noise; the difference between $3 \mathrm{GA}-\chi_{0}$ and $3 \mathrm{GA}-\chi_{0}^{w}$ shows the impact of time correlations ("color") in the noise, and the comparison to the ssLNA provides information on the importance of memory.

By way of a first orientation, Fig. 6 shows that the full 3GA model reduction gives results that are very close to the full dynamics both for individual trajectories [Figs. 6(a) and 6(b)] and for timedependent mean trajectories and variances [Figs. 6(d) and 6(e)]. The ssLNA [Fig. 6(c)] shows significant deviations, which can be attributed both to its neglect of memory [which is important for the means, Fig. 6(d)] and of time correlations in the extrinsic noise, resulting in variances being underestimated [Fig. 6(e)]. The quantification of these deviations in terms of single-species KL-divergence and covariance matrix accuracy [Figs. 7 (a) and 7(b)] confirms this. The 3GA- $\chi_{0}^{w}$ approximation gives similar accuracies as the ssLNA but is significantly better for short times as is visible in the KLdivergence values in Fig. 7(a). This is due to its inclusion of memory effects, which in previous studies ${ }^{12,13}$ were shown to give approximation errors that were orders of magnitude lower than for memoryless approximations such as the ssLNA. At long times, where the system has essentially reached its steady state, memory becomes unimportant and the $3 \mathrm{GA}-\chi_{0}^{w}$ and ssLNA perform similarly.
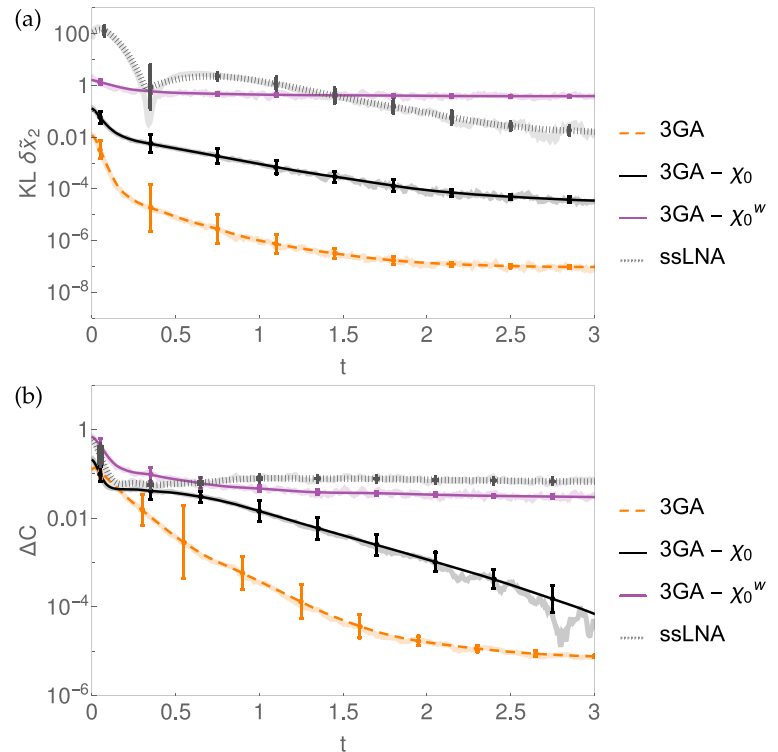

FIG. 7. Accuracy of effective subnetwork noise approximations at $\varepsilon=0.1$. (a) $\mathrm{KL}$ of subnetwork boundary species $\delta \tilde{x}_{2}$ and (b) error $\Delta C$ for the $3 \mathrm{GA}$, ssLNA, and intermediate approximations ( $3 \mathrm{GA}-\chi_{0}$ and $\left.3 \mathrm{GA}-\chi_{0}^{\mathrm{w}}\right)$, estimated from a sample of 1000 noise realizations $(\varepsilon=0.1)$. Smoothed curves with corresponding error bars are shown in dark color in addition to the raw numerical data (light color). The full $3 \mathrm{GA}$ reduction works best, although neglecting nonlinear noise effects (3GA- $\left.\chi_{0}\right)$ still gives a good approximation (small KL values).
The $3 \mathrm{GA}-\chi_{0}$ approximation, which keeps track of time correlations in the extrinsic noise, is significantly more accurate than the "white" approximations (ssLNA and 3GA- $\chi_{0}^{w}$ ). Its errors, as shown in Fig. 7, are clearly still larger than those of the full 3GA but remain small in quantitative terms [for a comparison with the corresponding Wasserstein distances, which lead to the same conclusion, see Fig. S1(c)]. We can thus summarize by saying that accurate subnetwork reductions require at least memory terms to make reasonable predictions for average time courses; for quantitative fluctuation estimates, time correlations in the extrinsic noise are also important, while nonlinear (multiplicative) features of this noise can be neglected without seriously impairing accuracy.

We also tested the accuracy of the various approximations when noise and random initial conditions in the bulk are both present (see Fig. S6). The initial bulk uncertainty can then-and does in our simple network example-become a significant driver of fluctuations in the dynamics. This makes the different treatment of the stochastic noise in the $3 \mathrm{GA}, 3 \mathrm{GA}-\boldsymbol{\chi}_{0}$, and $3 \mathrm{GA}-\boldsymbol{\chi}_{0}^{w}$ less important, with all three methods giving low errors. Only the ssLNA continues to perform poorly because it assumes that the bulk is always at steady state and so cannot capture initial bulk fluctuations.

\section{B. Variation with strength $\varepsilon$ of stochastic fluctuations}

The parameter $\varepsilon$ we have introduced sets the amplitude of stochastic fluctuations and plays a role complementary to $\varepsilon_{0}$, which controls the amplitude of deviations from the steady state in the bulk at the initial time. As a complement to the analysis in Sec. III D, where we varied $\varepsilon_{0}$ at $\varepsilon=0$, we now assess how the accuracy of the $3 \mathrm{GA}$, ssLNA, $3 \mathrm{GA}-\chi_{0}$, and $3 \mathrm{GA}-\chi_{0}^{w}$ varies with $\varepsilon$ when we set $\varepsilon_{0}=0$. As before, we set the subnetwork initial conditions to be at steady state to avoid competing error contributions. For each approximation, the error on the means scales as the first order in $\varepsilon$ not systematically accounted for; thus, one has (as discussed in Appendix B) $\Delta \mu \sim \varepsilon^{2}$ for 3GA, $\Delta \mu \sim \varepsilon$ for 3GA- $\chi_{0}, 3 \mathrm{GA}-\chi_{0}^{w}$, and ssLNA. For errors on covariances, one finds $\overline{\Delta C} \sim \varepsilon^{2}$ (3GA, 3GA- $\chi_{0}$ ) and $\overline{\Delta C} \sim \varepsilon$ (3GA- $\chi_{0}^{w}$ and ssLNA); for $\overline{\Delta \sigma^{2}}$, the same scalings hold. We confirm the validity of these power laws for our simple network model in Fig. 8. The errors of the ssLNA, in particular, are highest as expected, although in quantitative terms, they remain moderate. This arises from our use of steady state subnetwork initial conditions, which keep the exact mean concentrations close to steady state and hence to the ssLNA prediction (remaining exactly at steady state). In the general case, the ssLNA makes larger errors on the means, as shown by the large KL divergences in Fig. 7(a).

\section{EPIDERMAL GROWTH FACTOR RECEPTOR SIGNALING NETWORK}

The insights established by the previous analysis allow us to understand how to approximate the extrinsic noise, stemming both from random initial conditions in the unobserved bulk and the propagation of stochastic fluctuations, in biochemical networks. We now apply these insights to a key network around the Epidermal Growth Factor Receptor (EGFR). In this network, copy numbers of the relevant molecular species are large ${ }^{33,34}$ and stochastic fluctuations are therefore modest per se. As a consequence of this, attention has focused on the role of the cellular context in shaping heterogeneous 

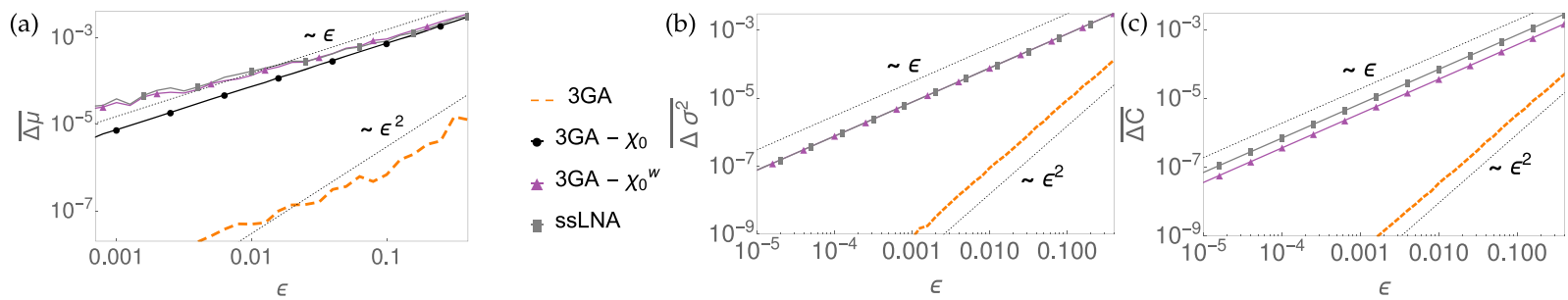

FIG. 8. Variation with $\varepsilon$ of integrated error: subnetwork means (a), variances (b), and correlations (c) over a sample of 2500 noise realizations for $3 G A$, ssLNA, and intermediate approximations of 3GA. Dotted lines show the theoretically expected power laws (see Appendix B). Initial conditions in the subnetwork are at steady state, $\delta \mathbf{x}^{\mathrm{s}}(0)=0$. For the means in (a), we use 3500 realizations of stochastic trajectories to suppress sampling errors, which are more significant here and mean we cannot meaningfully access noise levels $\varepsilon<0.001 \sim 1 / n$. (The deviations that are visible at low $\varepsilon$ arise from finite sample size errors on the means, which scale linearly with $\varepsilon$.) For the same reason, we do not show results for the $3 \mathrm{GA}-\chi_{0}$ approximation in (b) and (c).

outcomes of EGFR signaling. For instance, the observed heterogeneity of EGF dose-response curves for downstream processes such as the translocation of Extracellular signal-Regulated Kinases (ERK) to the nucleus ${ }^{35}$ and Ras signaling ${ }^{36}$ has been proposed to arise from cell-to-cell variation of signaling proteins such as EGFR and of other species involved in the signaling pathway.

We consider the model by Kholodenko et al., ${ }^{37}$ which describes the time evolution of 29 species involved in the EGFR signaling pathway. Following Ref. 12, we choose a subnetwork of 19 species, including Epidermal Growth Factor (EGF) and EGFR, which trigger the signaling reactions, and a bulk of 10 species, namely, Shc (Src homology and collagen domain protein) and all its complexes. The four subnetwork boundary species, directly affected by the interaction with the bulk, are SOS (Son of Sevenless homolog protein), Grb2 (growth factor receptor-binding protein 2), RP (tyrosine phosphorylated EGFR), and GS (Grb2-SOS complex); example results for their average time evolution and their variance around this are shown in Fig. 9.

In the EGFR network as modeled in Ref. 37, steady state concentrations $y_{i}$ range from $\sim 0.03$ to $\sim 600 \mathrm{nMol}$. If we estimate cells to have a diameter of $20 \mu \mathrm{m}^{38}$ and hence a volume of order $(20 \mu \mathrm{m})^{3}$, this gives an absolute steady state number of molecules $V y_{i}$ in a reaction compartment of volume $V$ in the range $\sim 140$ to $\sim 3 \cdot 10^{6}$, satisfying the criterion $V y_{i} \gg 1$ for our Gaussian initial condition approximation and the treatment by a CLE. We use units where the smallest $y_{i}$ is order unity and so choose a fluctuation amplitude $\varepsilon=0.01$ that correctly reproduces the smallest copy numbers $y_{i} / \varepsilon \sim 100$

We first study the accuracy of subnetwork equations with initial bulk uncertainty only, where we can apply both the path integral and projection approaches. We set $\varepsilon_{0}=0.01$ as just estimated. As the computational cost of the EGFR network is higher than for the simple network, we sample here 200 realizations of bulk initial conditions for the bulk and measure the accuracy of the full random force and 3GA effective noise and the different approximations to them. The quantitative results for the time-dependent KL divergences and $\Delta C$ are shown in Fig. S2 and confirm the general trends observed in the simple network: including the transient part $\overline{\Delta \boldsymbol{r}}$ of the extrinsic noise but simplifying it to an impulse at $t=0$ gives reasonable approximations. The persistent part $\boldsymbol{r}_{\infty}$ on its own yields low accuracy, and adding it to the transient $\overline{\Delta r}$ does not make a significant difference to predictions at short times. Looking at specific combinations of concentrations that feature in conservation laws does however reveal a non-negligible role of $\boldsymbol{r}_{\infty}$ in reproducing correlated fluctuations in the long-time steady state (see Appendix A and Sec. IV B of the supplementary material).

We next assess the performance of 3GA compared to the ssLNA and the linear-noise approximations of Sec. IV (and Table I) in the presence of (only) stochastic fluctuations of amplitude $\varepsilon=0.01$; the results are summarized in Fig. 10. In all these quantitative tests, we

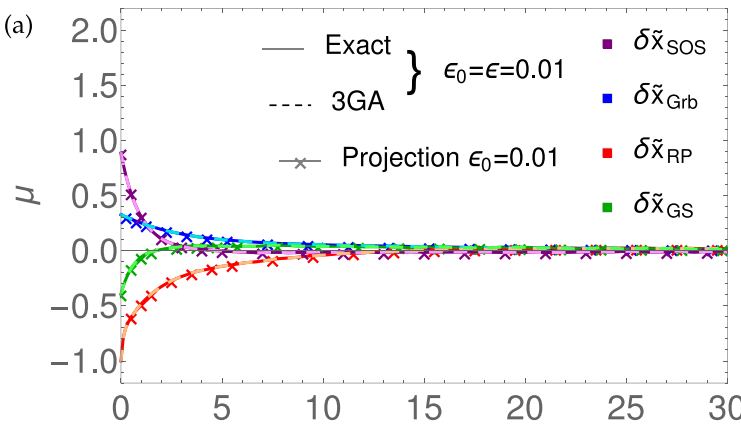

(b)

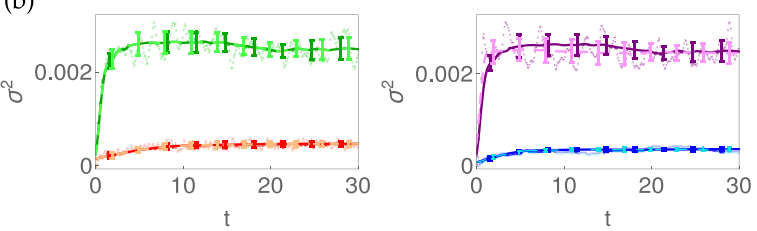

FIG. 9. Subnetwork dynamics in the EGFR network with intrinsic and extrinsic noise. (a) Temporal evolution of mean concentrations (normalized deviations from steady state) over 200 realizations of bulk random initial conditions and stochastic noise with $\varepsilon=\varepsilon_{0}=0.01$ (bold lines: exact and dashed lines: $3 G A$ ). Trajectories by projection methods represent conditional means for $\varepsilon \rightarrow 0$, and their average over the same random initial conditions (lines with crosses) is added as a comparison. (b) Comparison of variances, exact and 3GA, for the boundary species SOS, Grb, RP, and GS. Smoothing (with corresponding error bars) is applied and shown in dark color on top of numerical data (in light color). Here and in all EGFR plots, time is expressed in seconds. 
(a)
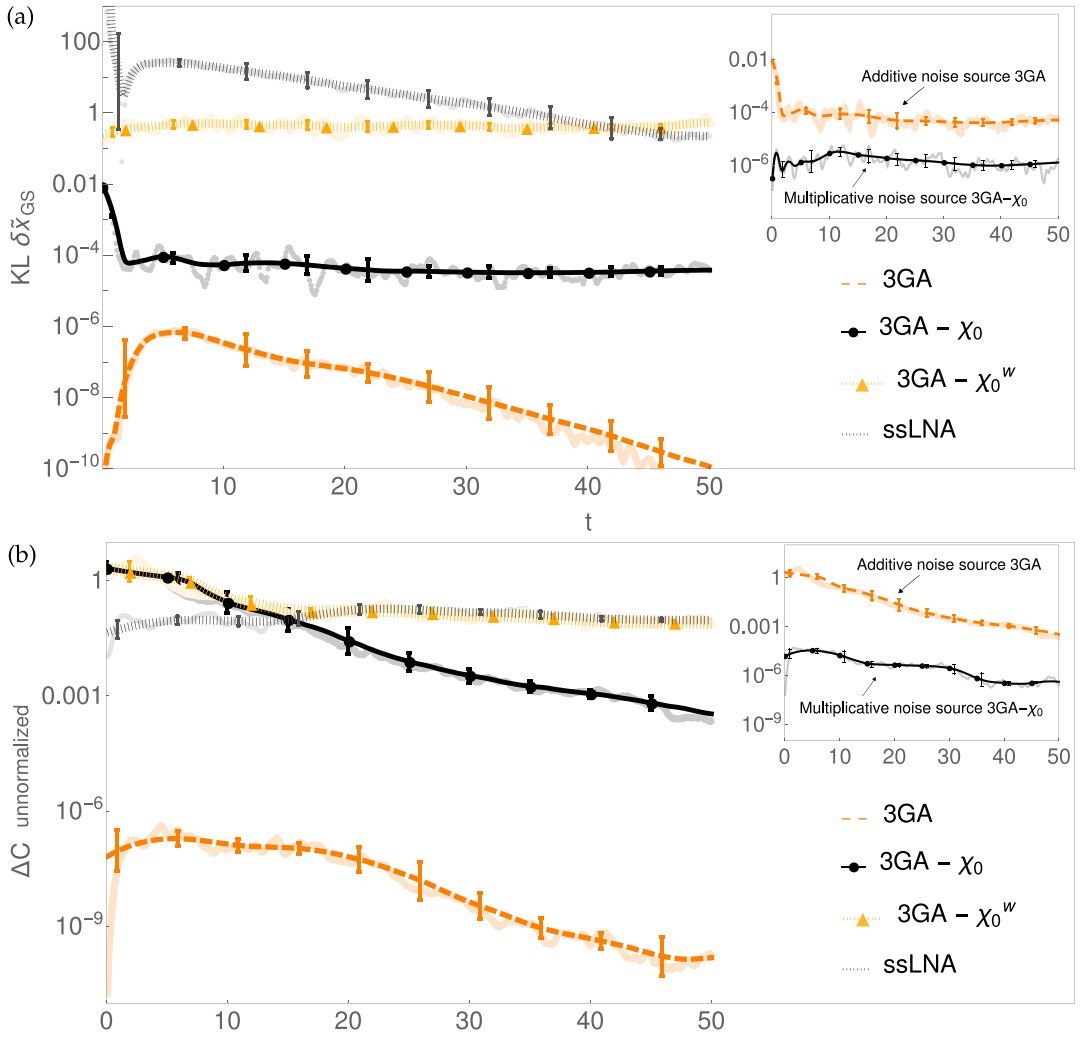

FIG. 10. Accuracy of effective subnetwork noise approximations at $\varepsilon=\mathbf{0 . 0 1}$. (a) $\mathrm{KL}$ for the boundary species $\mathrm{GS}$ and (b) covariance matrix error estimated on a sample of 200 realizations of white noise with $\varepsilon=0.01$ for $3 \mathrm{GA}$, intermediate approximations of $3 \mathrm{GA}\left(3 \mathrm{GA}-\chi_{0}\right.$ and $\left.3 \mathrm{GA}-\chi_{0}^{\mathrm{w}}\right)$, and ssLNA; the bulk is initially at steady state. The inset in (a) shows that the $K L$ for the $3 G A$ with an additive noise source is very similar to the one of $3 G A-\chi_{0}$; the inset in (b) shows that the same approximation gives a covariance error of $\mathrm{O}(1)$, like the other approximations where the noise is additive (3GA- $\chi_{0}$ and $\left.3 \mathrm{GA}-\chi_{0}^{w}\right)$; these approaches all underestimate the covariance on several subnetwork species. The insets in (a) and (b) also show that an improvement with respect to additive noise approximations is achieved by a linear noise approximation similar to $3 \mathrm{GA}-\chi_{0}$ if we neglect only the nonlinear propagation of effective noise but keep the multiplicative noise source (see Table I for details of this approximation). The covariance error in (b) was calculated without the normalization by steady state concentrations to avoid it becoming dominated by a few interior subnetwork species that have extremely low steady state concentrations and initially large deviations from this steady state (an example is shown in Fig. S5). For comparison, the version with normalized $\Delta C$ [as defined by (24)] is provided in supplementary material, Fig. S4.

have chosen challenging subnetwork initial conditions, as in Ref. 12, that maximize nonlinear effects in the subnetwork. This results in large initial values of the components of $\delta \tilde{\boldsymbol{x}}$ for some species. The linearization of $\sqrt{\boldsymbol{\Sigma}}$ in $\delta \boldsymbol{x}$ that we have used so far (see Sec. II of the supplementary material) then no longer makes sense, so we work with the full multiplicative noise $\boldsymbol{\xi}(t)$ with covariance (3) and (17) and define its nonlinear part as $\xi_{1}(t)=\xi(t)-\xi_{0}(t)$, · $=\mathrm{s}$, b. For more typical initial conditions closer to the steady state, we have verified that our standard linearization of $\sqrt{\Sigma}$ can be applied to the EGFR system without difficulties and gives sensible results.

The KL divergences resulting from the various approximations follow similar trends for the EGFR network model as in our simple network model; Fig. 10(a) shows data for one of the boundary species in the subnetwork. The same is true of the error on the covariance [Fig. 10(b)], with one exception: the ssLNA has lower errors in the initial transient here than the $3 \mathrm{GA}-\boldsymbol{\chi}_{0}$ and $3 \mathrm{GA}-\boldsymbol{\chi}_{0}^{w}$.
The reason is the accuracy with which correlations of interior subnetwork species are described, an effect that is not picked up by our single-species KL divergences (which have other benefits as explained above, specifically allowing us to focus on the memory effects that only affect boundary species). Specifically, some interior subnetwork species (mainly complexes formed by RP) are subject to strong relative fluctuations as they have low steady state concentrations but start from much larger initial values (see, for example, Fig. S5). The ssLNA, whose noise properties depend on the deterministic, fully nonlinear temporal trajectory, is able to reproduce these large fluctuations, while its rather rough treatment of effects from the bulk has a limited effect on interior subnetwork species. In contrast, our approximations with purely additive noise ( $3 \mathrm{GA}-\chi_{0}$ and $\left.3 \mathrm{GA}-\chi_{0}^{w}\right)$ underestimate these large interior subnetwork correlations, giving an error $\Delta C$ that is close to unity initially. 
This behavior allows us to further appreciate the role of the different terms that make up the nonlinear (multiplicative) contribution to the effective noise $\chi_{1}(t)(19)$ as predicted by the 3GA. It consists of (i) a multiplicative noise generated by nonlinear subnetwork-bulk reactions [i.e., the $\boldsymbol{\lambda}$-dependent terms of (19)] and (ii) a multiplicative noise source [i.e., terms $\xi_{1}^{\text {s }}$ and $\xi_{1}^{\mathrm{b}}$ of (19)]. We have tested what accuracy is achieved if we neglect either (i) or (ii): in the former case, we are effectively implementing a $3 \mathrm{GA}-\chi_{0}$ with multiplicative noise source, while in the latter case, we obtain a 3GA with additive noise source. Neglecting (i) has an impact only on boundary species (expected to make up a small fraction of large networks, e.g., here we have a subnetwork of 19 species, of which only 4 are boundary species), and we have found that indeed this keeps errors very small, as shown by the curves labeled "multiplicative noise source $3 \mathrm{GA}-\boldsymbol{\chi}_{0}$ " in the insets of Fig. 10. On the other hand, as soon as (ii) is neglected, which affects how well the fluctuations of every subnetwork species are captured, the accuracy of the 3GA becomes essentially the same as the $3 \mathrm{GA}-\boldsymbol{\chi}_{0}$ (curves labeled "additive noise source 3GA"). From these numerical tests, we can conclude that the improvement in the accuracy of the $3 \mathrm{GA}$ over the $3 \mathrm{GA}-\chi_{0}$ in modeling the EGFR system results mainly from accounting for the multiplicative nature of the noise sources.

Finally, we consider the full reaction equations including all stochastic terms, i.e., both intrinsic noise of amplitude $\varepsilon$ and fluctuating bulk initial conditions of amplitude $\varepsilon_{0}$. As both types of fluctuations arise from the same finite reaction volume, we take them equal, $\mathcal{E}=\varepsilon_{0}=0.01$. Averaging over multiple realizations of noise and randomly sampled bulk initial conditions, one obtains true conditional (on the initial subnetwork state) averages of subnetwork concentrations, which can be compared to the predictions from the projected equations for the same bulk initial conditions. These are approximate not only in their treatment of the random force [by truncating after terms of order $\left.\left(\delta x^{\mathrm{b}}\right)^{2}\right]$ but also via the fact that the memory functions used are calculated for $\varepsilon \rightarrow 0$ (see Ref. 12). Nonetheless, the agreement with the exact averages and those predicted by the $3 \mathrm{GA}$ method is excellent [see Fig. 9(a)]. Total variances can be estimated only in the 3GA approach, which yields extremely accurate results, as shown in Fig. 9(b). In the EGFR network, initial bulk uncertainty is not the main contribution to the overall effective noise as it was for the simple network model; hence, the comparison of the performance between the 3GA and the other approximations from Table I is essentially the same as in the case where only stochastic noise is present, as we show in Fig. S7.

\section{DISCUSSION}

In this paper, we have considered stochastic effects in mathematical models of biochemical subnetworks embedded in an environment (the bulk). This setting allows us to study systematically the emergence of extrinsic noise acting on the subnetwork due to the presence of the bulk and to analyze the structure of the various contributions to this noise. The general strategy here is to remove, i.e., integrate out or "marginalize," the bulk degrees of freedom to arrive at a reduced subnetwork description. For dynamics linearized around a fixed point, this can be done explicitly, showing that the time evolution of subnetwork boundary species-those that interact directly with the bulk-acquires both memory terms and an extrinsic noise that is colored (time-correlated). The extrinsic noise consists of two contributions: the first arises from the propagation of stochastic fluctuations in the bulk. The second contribution, which we refer to as random force, comes from the unknown bulk initial conditions. This fundamental structure can be generalized to nonlinear equations by two methods of systematic model reduction, namely, projection methods ${ }^{12}$ and a path-based marginalization over the bulk ${ }^{13}$ (3GA). While in previous works ${ }^{12,13}$ we focused on memory terms, by assuming the bulk to be initially at steady state and considering vanishing intrinsic noise, we have in this study investigated the effects of the environment when it is not initially at steady state, and one has to account also for its intrinsic fluctuations.

We saw that when only randomness in bulk initial conditions is present, it is possible to approximate the random force appearing in the projected equations as the sum of a short time piece and a constant piece that capture its transient and persistent parts, respectively. The first contribution is thus simplified to an impulse at $t=0$ (a $\delta$-function) with amplitude given by the integral of the short-time random force. It has the simple effect of perturbing the subnetwork initial conditions randomly. Adding the time-persistent piece gives what we called the "impulse plus persistent" approximation. It gives accurate results, with the transient piece being essential and the persistent piece giving smaller corrections. Analogous approximations for the colored noise appearing in the path integral 3GA approach lead to the same trends.

We next considered the fully stochastic case. To include intrinsic noise, one needs to step away from the projection approach, with its focus on conditional averages; we then use instead the path integral technique $3 \mathrm{GA}$, which can track all sources of fluctuations in the time evolution. Accordingly, there are two separate variances: one $\left(\varepsilon_{0}\right)$ for the initial bulk fluctuations and another $(\varepsilon)$ for the stochastic noise affecting all chemical reactions. We analyzed the scalings of errors with both parameters and found them to follow similar power laws, although their relative impact on the accuracy of predictions for the subnetwork dynamics depends on details of the reaction network such as reaction rates. [Note that for $\varepsilon>0$, relative concentration fluctuations cannot in general be made smaller than $O(\varepsilon)$, even for mean field-like situations where each molecular species takes part in many reactions; approaches based on linearization in fluctuations therefore always remain approximate to some extent, except in the joint limit of $\varepsilon \rightarrow 0$ and $\varepsilon_{0} \rightarrow 0$.]

We compared the 3GA approach with simplifications treating the extrinsic noise as linear (additive) or white linear (additive and without time correlations) and with the steady-state linear noise approximation (ssLNA); the latter treats all bulk dynamics as effectively instantaneous. We assessed the accuracy of these approximations for a simple network model and for the EGFR network and found that retaining in the subnetwork-reduced dynamics the time correlations ("color") of the extrinsic noise and its multiplicative (subnetwork-state dependent) nature is more important than nonlinear corrections. The time correlations, in particular, proved to be essential for properly reproducing the amplitude and temporal variation of correlations induced by extrinsic noise. The best choice of approximation for the extrinsic noise may be system-dependent: if there are transients where some concentrations that affect the colored noise covariance are far from steady state, as for the EGFR network, then any approximation that disregards the multiplicative 
nature of the noise source will be poor, regardless of the nonlinearities it accounts for, while relatively simple approximations such as the ssLNA can perform well. By contrast, if there are other dominant sources of variation such as the initial bulk uncertainty in our simple network model, then the focus needs to be on capturing these properly, while for the stochastic fluctuations, one can use approximations that are simpler than the full 3GA. An important question that would deserve future investigation is whether one can find a systematic strategy to assess the expected magnitude of the different contributions to the reduced dynamics starting from the rates and structure of the reaction network.

Several recent studies have concentrated on distinguishing sources of noise by conditioning on some part of the network in such a way as to split the fluctuations of some biochemical species into intrinsic contributions and those coming from either a static ${ }^{14,39}$ or dynamic ${ }^{40}$ environment, which also contribute to memory-like terms. ${ }^{41,42}$ Our expression for the colored noise [(12) and (19)] shows explicitly the decomposition of stochasticity into different contributions, first into noise intrinsic to the subnetwork and extrinsic noise, due to the interaction with the environment. ${ }^{14,43,44}$ A key aspect in our result is that we can further decompose the extrinsic noise into separate contributions coming from the random fluctuations inherent to biochemical reactions in the bulk and the uncertainty in its initial state. Our derivation of this decomposition is general (for unary and binary reactions, up to the second order) and fully retains dynamical features such as the transients in the time courses before a steady state is reached. Our result therefore generalizes previous steady state analyses ${ }^{15,45}$ to dynamics, without any $a$ priori assumption on the timescale of environmental fluctuations; the fast bulk approximation is recovered as a particular case. The tools to include other types of reactions in this framework, e.g., Michaelis-Menten enzyme reactions or gene regulation, are already available. ${ }^{46,47}$ Our approach to stochastic effects in subnetwork modeling allows us to understand to what extent variation in the system of interest is determined by fluctuations in the environment. If processes in the bulk can be parametrized and their rates can be estimated, at least within certain ranges, then our method enables one to put forward hypotheses on the sources of observed variability in the subnetwork. In this way, the properties of extrinsic noise can be deduced from statistical assumptions about the bulk dynamics, and these assumptions can then be refined by comparing the predicted and the measured effects on the subnetwork. For the sake of simplicity, we have limited the analysis to the Gaussian limit of Poisson fluctuation statistics for the bulk initial condition fluctuations, but the generality of (12) and (19) leaves open the possibility of considering different choices of fluctuating initial conditions. In addition, the strategy we have discussed in principle also applies to heterogeneity in bulk reaction rate constants. For mass-action kinetics, this can be treated on the same footing as fluctuations in bulk species by including the relevant rates into the state-space of the process as "virtual" bulk variables. This equivalence has been exploited to capture within the same mathematical framework extrinsic factors consisting either of heterogeneous, time-invariant reaction parametersakin to reaction rates in the bulk ${ }^{48,49}$ - or of temporally fluctuating variables-akin to bulk species. ${ }^{4}$

We have taken the EGFR network, whose structure and rates are well-characterized, as an illustrative example of how our mathematical methods can capture the dynamic propagation of noise as well as the persistent and transient contributions. Here, we have chosen the bulk to be the protein Src homology and collagen domain protein (Shc) and any complexes that include Shc, but one could straightforwardly repeat this analysis for other network splits, e.g., allocating to the bulk the extracellular stimuli that induce EGF binding, EGFR conformational changes, and phosporylation events, with in the subnetwork a selected set of reactions that are activated downstream. Among the targets, one could consider molecular species that are crucial to cell-fate decisions, such as SOS, Grb2 as well as Ras, MAPK (Mitogen-Activated Protein Kinase), and Erk; recent investigations highlight these as molecular species that are sensitive to cellular context variability. ${ }^{35,36,50}$ In this way, our mathematical framework could be applied to study how the variability in external signals and in the abundance of EGFR (induced, e.g., by downregulation, internalization, and subsequent degradation or recycling ${ }^{51}$ ) is conveyed by protein reaction cascades along the pathway to ultimately affect cellular responses to signaling. The linear scaling of $3 \mathrm{GA}$ reduction with the size of the bulk (see the discussion in Appendix D) enables one to deal with the high combinatorial complexity of protein interaction networks more efficiently than projection methods (which scale quadratically with bulk size). This would be important, e.g., for studying a more recent version of Kholodenko's EGFR signaling model ${ }^{52}$ that contains a significantly increased number of molecular species (over 300) by incorporating more details on protein domains and on feasible biochemical reactions. The example of heterogeneity in EGFR response hence sets the stage for a systematic use of our model reduction schemes, which can be flexibly adjusted to the theoretical or experimental question under investigation.

\section{SUPPLEMENTARY MATERIAL}

See the supplementary material for Secs. I-V and Figs. S1-S7.

\section{ACKNOWLEDGMENTS}

The authors acknowledge helpful discussions with Ramon Grima, Edgar Herrera-Delgado, and Brian Munsky.

\section{APPENDIX A: APPROXIMATION “IMPULSE PLUS PERSISTENT" OF THE RANDOM FORCE}

To understand in more detail what properties the random force in the full projection approach should have, we go back to the subnetwork equations for the concentration deviations in the subnetwork, denoted collectively by $\delta x^{s}$. For simplicity, we shall focus on the case of linearized dynamics at $\varepsilon \rightarrow 0$, written in the general form as

$$
\frac{d}{d t} \delta \boldsymbol{x}^{\boldsymbol{s}^{\mathrm{T}}}=\delta \boldsymbol{x}^{\boldsymbol{s}^{\mathrm{T}}} \boldsymbol{L}^{\mathrm{sS}}+\int_{0}^{t} d t^{\prime} \delta \boldsymbol{x}^{\mathrm{T}^{\mathrm{T}}} \boldsymbol{M}^{\mathrm{sS}}\left(t-t^{\prime}\right)+\boldsymbol{r}_{0}^{\mathrm{T}},
$$

where $\boldsymbol{r}_{0}(t)$ is given by (15). To write down the solution of the projected equations in the presence of nonzero random forces, we can introduce the Green's function $\boldsymbol{U}(t)$ for system (A1), which is determined by the rate matrix and memory function. In terms of this Green's function, the subnetwork concentrations at time $t$ are simply

$$
\delta \boldsymbol{x}^{\boldsymbol{s}^{\mathrm{T}}}(t)=\int_{0}^{t} d t^{\prime} \boldsymbol{r}_{0}^{\mathrm{T}}\left(t^{\prime}\right) \boldsymbol{U}\left(t-t^{\prime}\right)+\delta \boldsymbol{x}^{\mathrm{s}^{\mathrm{T}}}(0) \boldsymbol{U}(t) .
$$


The second term depends only on the subnetwork initial conditions and not on the random force, so we will ignore it in the following discussion. The convolution structure of $\delta x^{s^{T}}(t)$ suggests that for further analysis, one should split both $\boldsymbol{r}$ and $\boldsymbol{U}$ into two parts, one transient and one permanent, i.e., surviving to infinite time. Given again the convolution structure, it is convenient to work with the Laplace transform

$$
\delta \hat{\boldsymbol{x}}^{\hat{\mathrm{T}}^{\mathrm{T}}}(z)=\hat{\boldsymbol{r}}_{0}(z)^{\mathrm{T}} \hat{\boldsymbol{U}}(z)
$$

where $\hat{\boldsymbol{U}}(z)$ is given by

$$
\hat{\boldsymbol{U}}(z)=\left(z-\boldsymbol{L}^{\mathrm{ss}}-\hat{\mathbf{M}}^{\mathrm{ss}}(z)\right)^{-1} .
$$

Decomposing $\hat{\boldsymbol{U}}(z)$ and $\hat{\boldsymbol{r}}(z)$ into the transient and permanent part, we write

$$
\begin{aligned}
\hat{\boldsymbol{r}}_{0}(z) & =\frac{1}{z} \boldsymbol{r}_{0 \infty}(z)+\Delta \hat{\boldsymbol{r}}_{0}(z), \\
\hat{\boldsymbol{U}}(z) & =\frac{1}{z} \boldsymbol{U}_{\infty}+\Delta \hat{\boldsymbol{U}}(z) .
\end{aligned}
$$

The variation of the steady state due to the random force is then found as

$$
\begin{aligned}
\delta \boldsymbol{x}^{\mathrm{s}^{\mathrm{T}}}(t \rightarrow \infty)= & \lim _{z \rightarrow 0} z \hat{\boldsymbol{r}}_{0}^{\mathrm{T}}(z) \hat{\boldsymbol{U}}(z) \\
= & \lim _{z \rightarrow 0} z\left(\frac{1}{z} \boldsymbol{r}_{0 \infty}(z)+\Delta \hat{\boldsymbol{r}}_{0}(z)\right)^{\mathrm{T}}\left(\frac{1}{z} \boldsymbol{U}_{\infty}+\Delta \hat{\boldsymbol{U}}(z)\right) \\
= & \lim _{z \rightarrow 0}\left(\frac{1}{z} \boldsymbol{r}_{0 \infty}^{\mathrm{T}} \boldsymbol{U}_{\infty}+\boldsymbol{r}_{0 \infty}^{\mathrm{T}} \Delta \hat{\boldsymbol{U}}(z)+\Delta \hat{\boldsymbol{r}}_{0}^{\mathrm{T}}(z) \boldsymbol{U}_{\infty}\right. \\
& \left.+z \Delta \hat{\boldsymbol{r}}_{0}^{\mathrm{T}}(z) \Delta \hat{\boldsymbol{U}}(z)+\mathscr{O}(z)\right) .
\end{aligned}
$$

We show below that the apparently divergent first term does not contribute, while the last term vanishes for $z \rightarrow 0$. In the two remaining finite contributions, the small $z$-limit gives

$$
\Delta \hat{\boldsymbol{r}}_{0}(z \rightarrow 0)=\int_{0}^{\infty} d t^{\prime} \Delta \boldsymbol{r}_{0}\left(t^{\prime}\right)=\int_{0}^{\infty} d t^{\prime}\left[\boldsymbol{r}_{0}\left(t^{\prime}\right)-\boldsymbol{r}_{0 \infty}\right] \equiv \overline{\Delta \boldsymbol{r}_{0}}
$$

and similarly $\Delta \hat{\boldsymbol{U}}(z \rightarrow 0)=\overline{\Delta \boldsymbol{U}}$ so that

$$
\delta \boldsymbol{x}^{\mathrm{s}^{\mathrm{T}}}(t \rightarrow \infty)=\boldsymbol{r}_{0}^{\mathrm{T}} \overline{\Delta \boldsymbol{U}}+{\overline{\Delta \boldsymbol{r}_{0}}}^{\mathrm{T}} \boldsymbol{U}_{\infty} .
$$

This decomposition has two contributions, which are, respectively, the transient response to the persistent part of the random force and the persistent response to the transient part of the random force.

The analysis so far tells us that we will get the correct random force-dependent steady state from an approximate form of the random force as long as the approximation preserves both $\boldsymbol{r}_{0} \infty$ and $\overline{\Delta \boldsymbol{r}_{0}}$. Our proposal is then to construct the approximation by "concentrating" the transient part of the random force into a spike at $t=0$, specifically

$$
\boldsymbol{r}_{0}(t)=\boldsymbol{r}_{0 \infty}+\Delta \boldsymbol{r}_{0}(t) \simeq \boldsymbol{r}_{0 \infty}+\delta(t) \overline{\Delta \boldsymbol{r}_{0}} .
$$

Accordingly, we then approximate the linearized projected equations as

$$
\frac{d}{d t} \delta \boldsymbol{x}^{\mathrm{s}^{\mathrm{T}}}=\delta \boldsymbol{x}^{\mathrm{s}^{\mathrm{T}}} \boldsymbol{L}^{\mathrm{ss}}+\int_{0}^{t} d t^{\prime} \delta \boldsymbol{x}^{\mathrm{S}^{\mathrm{T}}} \boldsymbol{M}^{\mathrm{ss}}\left(t-t^{\prime}\right)+\boldsymbol{r}_{0 \infty}^{\mathrm{T}}+{\overline{\Delta \boldsymbol{r}_{0}}}^{\mathrm{T}} \delta(t) .
$$

Integrating a small time interval around $t=0$ then shows that the effect of the $\delta$-piece of the random force is equivalent to a random change in the initial subnetwork conditions by an amount $\overline{\Delta \boldsymbol{r}_{0}}$.

To see how the above approximation is implemented in practice, we recall that in terms of the subblocks of the matrix $\boldsymbol{L}$ representing the adjoint Fokker-Planck matrix operator, the random force is written as (15), i.e.,

$$
\boldsymbol{r}_{0}^{\mathrm{T}}(t)=\delta \boldsymbol{x}^{\mathrm{b}}(0)^{\mathrm{T}} e^{\boldsymbol{L}^{\mathrm{bb} t}} \boldsymbol{L}^{\mathrm{bs}} .
$$

If $\bar{\rho}$ and $\bar{l}$ are the right and left eigenvectors and $\mu$ are the eigenvalues of the matrix $L^{\mathrm{bb}}$, we can write the persistent and transient components of the random force explicitly as

$$
\begin{gathered}
\boldsymbol{r}_{0 \infty}^{\mathrm{T}}=\delta \boldsymbol{x}^{\mathrm{b}^{\mathrm{T}}}(0) \sum_{\beta, \mu_{\beta}=0} \overline{\boldsymbol{\rho}}_{\beta} \overline{\boldsymbol{l}}_{\beta}^{\mathrm{T}} \boldsymbol{L}^{\mathrm{bs}}, \\
{\overline{\Delta \boldsymbol{r}_{0}}}^{\mathrm{T}}=-\delta \boldsymbol{x}^{\mathrm{b}^{\mathrm{T}}}(0) \sum_{\beta, \mu_{\beta} \neq 0} \frac{1}{\mu_{\beta}} \overline{\boldsymbol{\rho}}_{\beta} \overline{\boldsymbol{l}}_{\beta}^{\mathrm{T}} \boldsymbol{L}^{\mathrm{bs}} .
\end{gathered}
$$

We can now use the eigenvector structure of $\boldsymbol{r}_{0 \infty}$ and $\boldsymbol{U}_{\infty}$ to show that the first term in (A6) vanishes. Let $\rho^{\mathrm{s}}$ and $\boldsymbol{l}^{\mathrm{s}}$ be the subnetwork components of the right and left eigenvectors of the full matrix $\boldsymbol{L}$ and $\lambda$ be the corresponding eigenvalues. Then, we can decompose $\hat{U}(z)(\mathrm{A} 4)$ into

$$
\hat{\boldsymbol{U}}=\sum_{\alpha, \lambda_{\alpha}=0} \frac{1}{z} \boldsymbol{\rho}_{\alpha}^{\mathrm{s}} \boldsymbol{l}_{\alpha}^{\mathrm{sT}}+\sum_{\alpha, \lambda_{\alpha} \neq 0} \frac{1}{z-\lambda_{\alpha}} \boldsymbol{\rho}_{\alpha}^{\mathrm{s}} \boldsymbol{l}_{\alpha}^{\mathrm{s}},
$$

from which one reads off

$$
\begin{aligned}
& \boldsymbol{U}_{\infty}=\sum_{\alpha, \lambda_{\alpha}=0} \boldsymbol{\rho}_{\alpha}^{\mathrm{s}} \boldsymbol{l}_{\alpha}^{\mathrm{s}}, \\
& \Delta \hat{\boldsymbol{U}}=\sum_{\alpha, \lambda_{\alpha} \neq 0} \frac{1}{z-\lambda_{\alpha}} \boldsymbol{\rho}_{\alpha}^{\mathrm{s}} \boldsymbol{l}_{\alpha}^{\mathrm{sT}} .
\end{aligned}
$$

Then, we can write $\boldsymbol{r}_{0 \infty}^{\mathrm{T}} \boldsymbol{U}_{\infty}$ using (A12) and (A14) as

$$
\begin{aligned}
\boldsymbol{r}_{0 \infty}^{\mathrm{T}} \boldsymbol{U}_{\infty} & =\delta \boldsymbol{x}^{\mathrm{b}^{\mathrm{T}}}(0) \sum_{\beta, \mu_{\beta}=0} \sum_{\alpha, \lambda_{\alpha}=0} \overline{\boldsymbol{\rho}}_{\beta} \overline{\boldsymbol{l}}_{\beta}^{\mathrm{T}} \boldsymbol{L}^{\mathrm{bs}} \boldsymbol{\rho}_{\alpha}^{\mathrm{s}} \boldsymbol{l}_{\alpha}^{\mathrm{s}^{\mathrm{T}}} \\
& =-\delta \boldsymbol{x}^{\mathrm{b}^{\mathrm{T}}}(0) \sum_{\beta, \mu_{\beta}=0} \sum_{\alpha, \lambda_{\alpha}=0} \overline{\boldsymbol{\rho}}_{\beta} \overline{\boldsymbol{l}}_{\beta}^{\mathrm{T}} \boldsymbol{L}^{\mathrm{bb}} \boldsymbol{\rho}_{\alpha}^{\mathrm{b}} \boldsymbol{l}_{\alpha}^{\mathrm{s}^{\mathrm{T}}} \\
& =0
\end{aligned}
$$

where the last line follows because $\overline{\boldsymbol{l}}_{\beta}^{\mathrm{T}} \boldsymbol{L}^{\mathrm{bb}}=0$ when $\overline{\boldsymbol{l}}_{\beta}$ is an eigenvector with zero eigenvalue of $\boldsymbol{L}^{\mathrm{bb}}$. We have also used the fact that $\boldsymbol{L}^{\mathrm{bs}} \boldsymbol{\rho}_{\alpha}^{\mathrm{s}}+\boldsymbol{L}^{\mathrm{bb}} \boldsymbol{\rho}_{\alpha}^{\mathrm{b}}=0$ for any right eigenvector of $\boldsymbol{L}$ with eigenvalue $\lambda_{\alpha}=0$; this identity allows one to eliminate $\boldsymbol{L}^{\mathrm{bs}}$ in favor of $\boldsymbol{L}^{\mathrm{bb}}$ in (A15). Note that $\left(-\boldsymbol{L}^{\text {ss }}-\boldsymbol{M}^{\text {ss }}\right)$ becomes non-invertible when we consider conserved quantities, and this occurs precisely when the response has a persistent part $\boldsymbol{U}_{\infty}$; therefore, the persistent part of the random force originates from conservation laws (see Sec. IV B of the supplementary material for an illustration in the context of the EGFR network). 
Motivated by the above analysis, we extend approximation (A9) to the random force $\boldsymbol{r}(t)=\boldsymbol{r}_{0}(t)+\boldsymbol{r}_{1}(t)$ of the nonlinear dynamics [to get (25)] as well as to the colored noise $\chi(t)=\chi_{0}(t)+\chi_{1}(t)$ of the 3GA-equations in the limit $\varepsilon \rightarrow 0$ (26). Disentangling the purely $\delta x^{\mathrm{b}}(0)$-dependent terms from the memory is not completely obvious from the mathematical derivation itself of the 3GA (see Sec. II of the supplementary material) and is easiest to understand from a numerical point of view, as explained in Appendix D 1.

\section{APPENDIX B: SCALINGS OF STATISTICS WITH $\varepsilon_{0}$ AND $\varepsilon$}

In this appendix, we assess how errors (27)-(29) scale with $\varepsilon_{0}$, i.e., with the variance of the initial bulk fluctuations, and with $\varepsilon$, i.e., the variance of stochastic fluctuations in the dynamics. To estimate this theoretically, we need to expand the solution of 3GA and projection equations in $\varepsilon_{0}$ and $\varepsilon$, which enter via $\delta \boldsymbol{x}^{\mathrm{b}}(0) \sim \sqrt{\varepsilon_{0}}$ and $\xi \sim \sqrt{\varepsilon}$, and see to what order the 3GA and projection methods are systematic.

We first focus on the 3GA path integral approach, which includes both dependencies. The subnetwork-reduced equation of motion (18), accounting for $\delta \boldsymbol{x}^{\mathrm{b}}(0)$ and $\boldsymbol{\xi}$ through $\boldsymbol{\chi}$ (12) and (19), has the schematic form

$$
\frac{d}{d t} \delta x^{\mathrm{s}}=\delta \boldsymbol{x}^{\mathrm{s}}+\delta \boldsymbol{x}^{\mathrm{s} 2}+\boldsymbol{\xi}+\delta \boldsymbol{x}^{\mathrm{s}} \boldsymbol{\xi}+O\left(\delta \boldsymbol{x}^{\mathrm{s} 3}, \delta \boldsymbol{x}^{\mathrm{s} 2} \boldsymbol{\xi}\right),
$$

where all terms are in general convolutions (e.g., the memory terms), but for simplicity, we do not write these explicitly. To start with, we shall also assume zero subnetwork initial conditions, $\delta x^{s}(0)=0$. The logic of the 3GA (Sec. II of the supplementary material) consists of keeping only the linear term on the rhs of (B1) initially, solving with Green's functions, and then treating the other terms perturbatively. In this way, $\delta x^{\mathrm{s}}$ is expressed as an expansion in $\delta x^{\mathrm{b}}(0)$ and $\boldsymbol{\xi}$ up to second order (again involving time convolutions that we leave implicit), giving schematically a solution

$$
\delta \boldsymbol{x}^{\mathrm{s}}=\boldsymbol{\xi}+\boldsymbol{\xi}^{2}+\delta \boldsymbol{x}^{\mathrm{b}}(0)+\delta \boldsymbol{x}^{\mathrm{b} 2}(0)+\boldsymbol{\xi} \delta \boldsymbol{x}^{\mathrm{b}}(0) .
$$

This solution will be accurate up to the order written, i.e., all third order terms are unsystematic. To disentangle the dependence on $\varepsilon$ from the one on $\varepsilon_{0}$, let us set $\delta x^{\mathrm{b}}(0)=0$. If we consider that pure $\xi^{3}$ terms average out as $\xi$ is drawn from a Gaussian with zero mean [see Eq. (3)], (B2) gives an estimate of the absolute errors on the means as $\overline{\Delta \mu} \sim \xi^{4} \sim \varepsilon^{2}$. For the second moments, we take the square of (B2), showing that the first terms not systematically included are fourth order ones in $\xi$. The errors on correlations $\overline{\Delta C}$ and on the variance $\overline{\Delta \sigma^{2}}$ are thus also expected to scale as $\varepsilon^{2}$ in leading order.

We next look at errors within the simpler approximations. The 3 GA- $\chi_{0}$, which neglects $\chi_{1}(t)$, can be systematic only up to $O(\sqrt{\varepsilon})$, i.e., up to $\xi$ in the perturbative expansion (B2). This gives errors on means that are now $\overline{\Delta \mu} \sim \xi^{2} \sim \varepsilon$, while from the square of (B2), one estimates errors on second moments $\overline{\Delta C} \sim \overline{\Delta \sigma^{2}} \sim \varepsilon^{2}$. Approximations that take the noise as white, such as the $3 \mathrm{GA}-\chi_{0}^{w}$ and ssLNA, are no longer systematic at $O(\sqrt{\varepsilon})$ as they change the stochastic noise properties already at leading order, but as terms $O(\sqrt{\varepsilon})$ average out for Gaussian noise, one again gets $\overline{\Delta \mu} \sim \varepsilon$. The correlations and variances will be incorrect already to leading order, on the other hand, i.e., have errors $\sim \mathcal{E}$. The ssLNA, which retains nonlinearities only at the level of the deterministic dynamics and so actually gives an $\varepsilon$ independent prediction of the means, has the same error scalings as the $3 \mathrm{GA}-\chi_{0}^{w}$.

The same line of reasoning as above is valid for the scalings with $\varepsilon_{0}$, given the dependence on $\delta x^{\mathrm{b}}(0)$ in (B2). The dependence of $\delta x^{\mathrm{s}}$ from the projection approach on $\delta x^{\mathrm{b}}(0)$ is also the same, given that it is determined through the random force. [We recall that the projection method, as developed in Ref. 12 and summarized in Sec. I of the supplementary material, can predict the linear and quadratic contributions to the random force in the initial values $\delta x^{\mathrm{b}}(0)$, but not terms $\left.O\left(\delta x^{\mathrm{b}}(0)^{3}\right)\right]$. As a result, one obtains, for both 3GA and projection methods, the scalings $\overline{\Delta \mu} \sim \overline{\Delta C} \sim \overline{\Delta \sigma^{2}} \sim \varepsilon_{0}^{2}$.

Finally, the effect of nonzero initial conditions $\delta x^{\mathrm{s}}(0)$ in the subnetwork would be to generate additional deviations. For the mean values, these will scale as $O\left(\delta \boldsymbol{x}^{\mathrm{s}}(0)^{3}\right)$ and will dominate over the sources of error discussed above once $\varepsilon$ and $\varepsilon_{0}$ are sufficiently small.

\section{APPENDIX C: SIMPLE NETWORK MODEL}

The simple network model of Fig. 1 is composed of the following reactions:

$$
\begin{array}{r}
1+2 \underset{k_{12}^{-}}{\stackrel{k_{12}^{+}}{\rightleftarrows}} 12, \\
1+3 \underset{k_{13}^{-}}{\stackrel{k_{13}^{+}}{\rightleftarrows}} 13, \\
2+3 \underset{k_{23}^{-}}{\stackrel{k_{23}^{+}}{\rightleftarrows}} 23, \\
1 \underset{\lambda_{12}}{\stackrel{\lambda_{21}}{\rightleftarrows}} 2, \\
13 \underset{\lambda_{23}}{\stackrel{\lambda_{13}}{\rightleftarrows}} 23,
\end{array}
$$

which can be described by mass-action kinetics (1) and (2). $k_{12}^{+}, k_{13}^{+}$, and $k_{23}^{+}$are complex formation rate constants; $k_{12}^{-}, k_{13}^{-}$, and $k_{23}^{-}$are complex dissociation rates; and $\lambda_{12}, \lambda_{21}, \lambda_{13}$, and $\lambda_{23}$ are unary reaction rates. For our numerical examples, their values are set to $k_{12}^{+}=1$, $k_{12}^{-}=2, k_{13}^{+}=1, k_{13}^{-}=2, k_{23}^{+}=2, k_{23}^{-}=3, \lambda_{12}=0.5, \lambda_{21}=1, \lambda_{13}$ $=0.5$, and $\lambda_{23}=1$, which gives the steady state values $y_{1}=0.5, y_{2}=0.2$, $y_{12}=0.05, y_{3}=9.5, y_{13}=2.4$, and $y_{23}=1.25$.

\section{APPENDIX D: EFFECTIVE 3CA EQUATION SOLVER WITH EFFECTIVE NOISE}

In this appendix, we discuss the numerical solution of the 3GA subnetwork equations with effective noise from the bulk. For an analogous discussion on the numerical implementation of projected equations, we refer to Ref. 53. The numerical solution of the stochastic integro-differential equations (18) can be simplified by mapping every integral term into the solution of ordinary stochastic differential equations. The subnetwork reduced dynamics has the form

$$
\frac{d}{d t} \delta \boldsymbol{x}^{\mathrm{s}^{\mathrm{T}}}(t)=\delta \boldsymbol{x}^{\mathrm{s}^{\mathrm{T}}}(t) \boldsymbol{L}^{\mathrm{ss}}+\left(\delta \boldsymbol{x}^{\mathrm{s}^{\mathrm{T}}}(t) \circ \delta \boldsymbol{x}^{\mathrm{s}^{\mathrm{T}}}(t)\right) \boldsymbol{L}^{\text {ss,s }}+\mathscr{M}^{\mathrm{T}}(t)+\chi^{\mathrm{T}}(t)
$$


In the derivation of the effective noise $\chi(t)$, we have rewritten this quantity [see Eq. (19)] in terms of $\lambda(t)=\boldsymbol{E}_{\mathrm{bb}}^{\mathrm{T}}(t) \delta \boldsymbol{x}^{\mathrm{b}}(0)+\int_{0}^{t} d t^{\prime} \boldsymbol{E}_{\mathrm{bb}}^{\mathrm{T}}$ $\left(t-t^{\prime}\right) \xi_{0}^{\mathrm{b}}\left(t^{\prime}\right)$ [Eq. (21)], which is the overall linear noise coming out of the bulk, both from initial bulk fluctuations $\delta x^{\mathrm{b}}(0)$ and stochastic fluctuations $\xi^{\mathrm{b}}(t)$. Thus, to implement the colored noise, we need auxiliary equations also for $\lambda(t)$. In this process, it is expedient to combine $\lambda(t)$ with the contribution needed to implement the memory, $\boldsymbol{v}(t)$ (see Ref. 13 and Sec. II of the supplementary material). We define $\boldsymbol{g}_{0}(t)$ as $\boldsymbol{g}_{0}^{\mathrm{T}}(t)=\int_{0}^{t} d t^{\prime}\left[\delta \boldsymbol{x}^{\mathrm{s}^{\mathrm{T}}}\left(t^{\prime}\right) \boldsymbol{L}^{\mathrm{sb}}+\boldsymbol{\xi}_{0}^{\mathrm{b}^{\mathrm{T}}}\left(t^{\prime}\right)\right] \boldsymbol{E}_{\mathrm{bb}}\left(t-t^{\prime}\right)$ $+\delta \boldsymbol{x}^{\mathrm{b}^{\mathrm{T}}}(0) \boldsymbol{E}_{\mathrm{bb}}(t)=\boldsymbol{v}^{T}(t)+\boldsymbol{\lambda}^{T}(t)$ and $\boldsymbol{g}_{1}(t)$ as $\boldsymbol{g}_{1}^{\mathrm{T}}(t)=\int_{0}^{t} d t^{\prime} \boldsymbol{\xi}_{1}^{\mathrm{b}^{\mathrm{T}}}\left(t^{\prime}\right) \boldsymbol{E}_{\mathrm{bb}}$ $\left(t-t^{\prime}\right)$. Their sum $\boldsymbol{g}(t)=\boldsymbol{g}_{0}(t)+\boldsymbol{g}_{1}(t)=\boldsymbol{v}(t)+\boldsymbol{\lambda}(t)+\boldsymbol{g}_{1}(t)$ is a vector of size $N^{\mathrm{b}}$ (number of bulk species), which can be obtained by solving the $N^{\mathrm{b}}$ differential equations

$$
\frac{d \boldsymbol{g}^{\mathrm{T}}(t)}{d t}=\boldsymbol{g}^{\mathrm{T}}(t) \boldsymbol{L}^{\mathrm{bb}}+\delta \boldsymbol{x}^{\mathrm{s}^{\mathrm{T}}} \boldsymbol{L}^{\mathrm{sb}}(t)+\boldsymbol{\xi}_{0}^{\mathrm{b}^{\mathrm{T}}}(t)+\boldsymbol{\xi}_{1}^{\mathrm{b}^{\mathrm{T}}}(t)
$$

with initial condition $\boldsymbol{g}(0)=\delta \boldsymbol{x}^{\mathrm{b}}(0)$. The evolution of $\boldsymbol{g}$ is then the linearized bulk dynamics with an additive part $\left\langle\xi_{0}^{\mathrm{b}}(t) \xi_{0}^{\mathrm{b}^{\mathrm{T}}}\left(t^{\prime}\right)\right\rangle$ $=\Sigma_{0}^{\mathrm{bb}} \delta\left(t-t^{\prime}\right)$ and a multiplicative part $\left\langle\xi_{1}^{\mathrm{b}}(t) \xi_{1}^{\mathrm{b}^{\mathrm{T}}}\left(t^{\prime}\right)\right\rangle=\Sigma_{1}^{\mathrm{bb}}\left(\delta \boldsymbol{x}^{\mathrm{s}}, \delta \boldsymbol{x}^{\mathrm{b}}\right.$ $=\boldsymbol{g}) \delta\left(t-t^{\prime}\right)$. The non-Markovian part of the effective subnetwork equation (D1) can be written as

$$
\begin{aligned}
\mathscr{M}^{\mathrm{T}}(t)+\chi^{\mathrm{T}}(t)= & \boldsymbol{g}^{\mathrm{T}}(t) \boldsymbol{L}^{\mathrm{bs}}+\boldsymbol{\xi}_{0}^{\mathrm{s}^{\mathrm{T}}}(t)+\boldsymbol{\xi}_{1}^{\mathrm{s}^{\mathrm{T}}}(t)+\left(\delta \boldsymbol{x}^{\mathrm{s}}(t) \circ \boldsymbol{g}_{0}(t)\right)^{\mathrm{T}} \boldsymbol{L}^{\mathrm{sb}, \mathrm{s}} \\
& +\int_{0}^{t} d t^{\prime}\left[\left(\delta \boldsymbol{x}^{\mathrm{s}}\left(t^{\prime}\right) \circ \boldsymbol{g}_{0}\left(t^{\prime}\right)\right)^{\mathrm{T}} \boldsymbol{L}^{\mathrm{sb}, \mathrm{b}}\right. \\
& \left.+\left(\boldsymbol{g}_{0}\left(t^{\prime}\right) \circ \boldsymbol{g}_{0}\left(t^{\prime}\right)\right)^{\mathrm{T}} \boldsymbol{L}^{\mathrm{bb}, \mathrm{b}}\right] \boldsymbol{E}_{\mathrm{bb}}\left(t-t^{\prime}\right) \boldsymbol{L}^{\mathrm{bs}} .
\end{aligned}
$$

The $\boldsymbol{v}(t)$-part of $\boldsymbol{g}_{0}(t)$ contributes to the memory $\mathscr{M}(t)$ (as explained in Ref. 13), while the contributions from $\lambda(t)$ and $\boldsymbol{g}_{1}(t)$, along with the additional terms in $\xi_{0}^{\mathrm{T}^{\mathrm{T}}}(t)$ and $\xi_{1}^{\mathrm{s}^{\mathrm{T}}}(t)$, give rise to the subnetwork colored noise $\chi(t)$. The time integral left in the last term of (D3) can be evaluated by introducing ad hoc auxiliary variables, similar to the strategy adopted in Refs. 12 and 13. We diagonalize $\boldsymbol{L}^{\mathrm{bb}}$ and decompose the exponential kernel $\boldsymbol{E}_{\mathrm{bb}}\left(t-t^{\prime}\right)=e^{\boldsymbol{L}^{\mathrm{bb}}\left(t-t^{\prime}\right)}$ into a superposition of pure exponentials,

$$
\begin{aligned}
\int_{0}^{t} d t^{\prime} & {\left[\left(\delta \boldsymbol{x}^{\mathrm{s}}\left(t^{\prime}\right) \circ \boldsymbol{g}_{0}\left(t^{\prime}\right)\right)^{\mathrm{T}} \boldsymbol{L}^{\mathrm{sb}, \mathrm{b}}+\left(\boldsymbol{g}_{0}\left(t^{\prime}\right) \circ \boldsymbol{g}_{0}\left(t^{\prime}\right)\right)^{\mathrm{T}} \boldsymbol{L}^{\mathrm{bb}, \mathrm{b}}\right] e^{\boldsymbol{L}^{\mathrm{bb}}\left(t-t^{\prime}\right)} \boldsymbol{L}^{\mathrm{bs}} } \\
= & \int_{0}^{t} d t^{\prime}\left[\left(\delta \boldsymbol{x}^{\mathrm{s}}\left(t^{\prime}\right) \circ \boldsymbol{g}_{0}\left(t^{\prime}\right)\right)^{\mathrm{T}} \boldsymbol{L}^{\mathrm{sb}, \mathrm{b}}+\left(\boldsymbol{g}_{0}\left(t^{\prime}\right) \circ \boldsymbol{g}_{0}\left(t^{\prime}\right)\right)^{\mathrm{T}} \boldsymbol{L}^{\mathrm{bb}, \mathrm{b}}\right] \\
& \times \sum_{\beta=1}^{N^{\mathrm{b}}} \overline{\boldsymbol{\rho}}_{\beta} \overline{\boldsymbol{l}}_{\beta}^{\mathrm{T}} e^{\mu_{\beta}\left(t-t^{\prime}\right)} \boldsymbol{L}^{\mathrm{bs}},
\end{aligned}
$$

where $\mu_{\beta}$ are the $N^{\mathrm{b}}$ eigenvalues of $\boldsymbol{L}^{\mathrm{bb}}$ and $\overline{\boldsymbol{\rho}}_{\beta}$ and $\overline{\boldsymbol{l}}_{\beta}$ are, respectively, its right and left eigenvectors and give the coefficients of this decomposition. To evaluate the time integral, we can now solve a differential equation for each component of the vector $z(t)=\left\{z^{\beta}(t)\right\}$, $\beta=1, \ldots, N^{\mathrm{b}}$,

$$
\begin{aligned}
\frac{d}{d t} z^{\beta}(t)= & \mu_{\beta} z^{\beta}(t)+\left[\left(\delta \boldsymbol{x}^{\mathrm{s}}\left(t^{\prime}\right) \circ \boldsymbol{g}_{0}\left(t^{\prime}\right)\right)^{\mathrm{T}} \boldsymbol{L}^{\mathrm{sb}, \mathrm{b}}\right. \\
& \left.+\left(\boldsymbol{g}_{0}\left(t^{\prime}\right) \circ \boldsymbol{g}_{0}\left(t^{\prime}\right)\right)^{\mathrm{T}} \boldsymbol{L}^{\mathrm{bb}, \mathrm{b}}\right] \overline{\boldsymbol{\rho}}_{\beta}, \quad z^{\beta}(0)=0 .
\end{aligned}
$$

In terms of these auxiliary variables, we can finally rewrite (D1) as

$$
\begin{aligned}
\frac{d}{d t} \delta \boldsymbol{x}^{s^{\mathrm{T}}}(t)= & \delta \boldsymbol{x}^{\mathrm{s}^{\mathrm{T}}}(t) \boldsymbol{L}^{\mathrm{ss}}+\left(\delta \boldsymbol{x}^{\mathrm{s}}(t) \circ \delta \boldsymbol{x}^{\mathrm{s}}(t)\right)^{\mathrm{T}} \boldsymbol{L}^{\mathrm{ss}, \mathrm{s}}+\boldsymbol{g}^{\mathrm{T}}(t) \boldsymbol{L}^{\mathrm{bs}}+\boldsymbol{\xi}_{0}^{\mathrm{s}^{\mathrm{T}}}(t) \\
& +\boldsymbol{\xi}_{1}^{\mathrm{T}^{\mathrm{T}}}(t)+\left(\delta \boldsymbol{x}^{\mathrm{s}}(t) \circ \boldsymbol{g}_{0}(t)\right)^{\mathrm{T}} \boldsymbol{L}^{\mathrm{sb}, \mathrm{s}}+\sum_{\beta=1}^{N^{\mathrm{b}}} z^{\beta}(t) \overline{\boldsymbol{l}}_{\beta}^{\mathrm{T}} \boldsymbol{L}^{\mathrm{bs}} .
\end{aligned}
$$

As in Ref. 13, solving the $N^{\text {s }}$ subnetwork equations is equivalent to solving a system with $2 \mathrm{~N}^{\mathrm{b}}$ additional equations, $N^{\mathrm{b}}$ for $\boldsymbol{g}(t)$ [see Eq. (D2)] and $N^{\mathrm{b}}$ for $\boldsymbol{z}(t)$ [see Eq. (D5)]. For projection methods, ${ }^{12}$ on the other hand, the analogous reduction from memory integrals to differential equations requires a number of additional variables equal to the dimension of the bulk variable space with all the possible concentration products, which scales as $\left(N^{\mathrm{b}} \times N^{\mathrm{b}}\right)$ $+\left(N^{\mathrm{s}} \times N^{\mathrm{b}}\right)$.

\section{1. $\varepsilon_{0}$-component of $\chi(t)$.}

In the regime $\varepsilon \rightarrow 0$, i.e., where the extrinsic noise arises only from the uncertainty on bulk initial conditions, one uses only the $\delta \boldsymbol{x}^{\mathrm{b}}(0)$ part of $\boldsymbol{\lambda}(t)$ and sets $\boldsymbol{g}_{1}=0$ (so $\boldsymbol{g}=\boldsymbol{g}_{0}$ ). Next, the resulting $\chi(t)$ can then be further approximated by short-time and long-time parts (26) after having isolated the 3GA colored noise $\chi(t)$ from the memory. To do so, we solve the rhs of (D3) either with the full $\boldsymbol{g}(t)$ or with $\boldsymbol{g}(t)=\boldsymbol{v}(t)$ [i.e., without the sources of randomness $\delta \boldsymbol{x}^{\mathrm{b}}(0)$ appearing in $\lambda$ ] and we take as $\chi(t)$ the difference between these two solutions; note that in both of these solutions, $\delta x^{\mathrm{s}}(t)$ is evaluated at the nonzero $\delta x^{\mathrm{b}}(0)$.

\section{2. $\varepsilon$-component of $\chi(t)$.}

In the presence of stochastic fluctuations, one notes that the variables $\boldsymbol{g}$ and $\boldsymbol{g}_{0}$ appearing in (D6) become distinct. To avoid having to calculate these variables separately, one can approximate in one of two ways: (i) use $\boldsymbol{g}(t)$ everywhere, which gives the full 3GA with a multiplicative noise source but implicitly adds within the colored noise $\chi_{1}(t)$ some terms $\sim\left(\delta x^{s}\right)^{3 / 2}$ of higher order than we have kept, and (ii) use $\boldsymbol{g}_{0}(t)$ everywhere. The latter option is equivalent to dropping the multiplicative noise terms $\xi_{1}^{\mathrm{b}}$ in (D2). One should then consistently also neglect $\xi_{1}^{s}$ in (D6), and this defines what we have called the "3GA with additive noise source" in Table I.

The noise can be further approximated by either $3 \mathrm{GA}-\boldsymbol{\chi}_{0}$ or $3 \mathrm{GA}-\chi_{0}^{w}$ (see Sec. IV and Table I). To implement the former, we neglect $\xi_{1}^{s}$ in (D6) and introduce a new variable $\boldsymbol{g}_{0}^{*}$ evolving according to the deterministic version of (D2); we then replace $\boldsymbol{g}_{0}$ by $\boldsymbol{g}_{0}^{*}$ in the nonlinear terms in (D6) with coefficients $\boldsymbol{L}^{\text {sb,s }}$ and similarly in (D5) with coefficients $\boldsymbol{L}^{\mathrm{sb}, \mathrm{b}}, \boldsymbol{L}^{\mathrm{bb}, \mathrm{b}}$. This ensures that the nonlinear memory is preserved, but the effective noise appears only through linear terms $\boldsymbol{L}^{\mathrm{bs}}$. For the $3 \mathrm{GA}-\boldsymbol{\chi}_{0}^{w}$ approximation, we use the same set of equations where $\boldsymbol{g}_{0}^{*}$ appears also in the term multiplied by $\boldsymbol{L}^{\text {bs }}$ in (D6) so that propagation of noise from the bulk is excluded also in this linear term; instead, we explicitly add the linear white noise $\boldsymbol{\chi}_{0}^{w}$ from (30) to (D6). Without initial bulk fluctuations, one needs simply to drop the corresponding contributions to $\lambda$ and $\boldsymbol{g}_{0}$. 


\section{DATA AVAILABILITY}

The data that support the findings of this study are available from the corresponding author upon reasonable request.

\section{REFERENCES}

${ }^{1}$ M. Apri, M. De Gee, and J. Molenaar, "Complexity reduction preserving dynamical behavior of biochemical networks," J. Theor. Biol. 304, 16-26 (2012).

${ }^{2}$ J. Ackermann, J. Einloft, J. Nöthen, and I. Koch, "Reduction techniques for network validation in systems biology," J. Theor. Biol. 315, 71-80 (2012).

${ }^{3}$ O. Radulescu, A. Gorban, A. Zinovyev, and V. Noel, "Reduction of dynamical biochemical reactions networks in computational biology," Front. Genet. 3, 131 (2012).

${ }^{4}$ J. Paulsson, O. G. Berg, and M. Ehrenberg, "Stochastic focusing: Fluctuationenhanced sensitivity of intracellular regulation," Proc. Natl. Acad. Sci. U. S. A. 97, 7148-7153 (2000).

${ }^{5} \mathrm{M}$. Del Giudice, S. Bo, S. Grigolon, and C. Bosia, "On the role of extrinsic noise in microRNA-mediated bimodal gene expression," PLOS Computational Biology 14, e1006063 (2018)

${ }^{6}$ M. R. Birtwistle, J. Rauch, A. Kiyatkin, E. Aksamitiene, M. Dobrzyński, J. B. Hoek, W. Kolch, B. A. Ogunnaike, and B. N. Kholodenko, "Emergence of bimodal cell population responses from the interplay between analog singlecell signaling and protein expression noise," BMC Syst. Biol. 6, 109 (2012), http://www.biomedcentral.com/1752-0509/6/109.

${ }^{7}$ B. Bravi and G. Longo, "The unconventionality of nature: Biology, from noise to functional randomness," in Proceedings of the Unconventional Computation and Natural Computation Conference (UCNC), edited by C. Calude and M. J. Dinneen (Springer, 2015), pp. 3-34.

${ }^{8}$ M. S. Okino and M. L. Mavrovouniotis, "Simplification of mathematical models of chemical reaction systems," Chem. Rev. 98, 391-408 (1998).

${ }^{9}$ M. Sunnaker, G. Cedersund, and M. Jirstrand, "A method for zooming of nonlinear models of biochemical systems," BMC Syst. Biol. 5, 140 (2011).

${ }^{10} \mathrm{R}$. Zwanzig, "Memory effects in irreversible thermodynamics," Phys. Rev. 124, 983 (1961).

${ }^{11}$ F. Ritort and P. Sollich, "Glassy dynamics of kinetically constrained models," Adv. Phys. 52, 219-342 (2003).

${ }^{12}$ K. J. Rubin, K. Lawler, P. Sollich, and T. Ng, "Memory effects in biochemical networks as the natural counterpart of extrinsic noise," J. Theor. Biol. 357, 245267 (2014).

${ }^{13}$ B. Bravi and P. Sollich, "Statistical physics approaches to subnetwork dynamics in biochemical systems," Phys. Biol. 14, 045010 (2017).

${ }^{14}$ P. S. Swain, M. B. Elowitz, and E. D. Siggia, "Intrinsic and extrinsic contributions to stochasticity in gene expression," Proc. Natl. Acad. Sci. U. S. A. 99, 12795-12800 (2002).

${ }^{15} J$. Paulsson, "Summing up the noise in gene networks," Nature 427, 415-418 (2004).

${ }^{16}$ P. Thomas, R. Grima, and A. Straube, "Rigorous elimination of fast stochastic variables from the linear noise approximation using projection operators," Phys. Rev. E 86, 041110 (2012).

${ }^{17}$ S. Bo and A. Celani, "Multiple-scale stochastic processes: Decimation, averaging and beyond," Phys. Rep. 670, 1-59 (2017).

${ }^{18} \mathrm{P}$. Chvykov and J. England, "Least-rattling feedback from strong time-scale separation," Phys. Rev. E 97, 032115 (2018).

${ }^{19}$ N. A. Sinitsyn, N. Hengartner, and I. Nemenman, "Adiabatic coarse-graining and simulations of stochastic biochemical networks," Proc. Natl. Acad. Sci. U. S. A. 106, 10546-10551 (2009).

${ }^{20} \mathrm{P}$. Thomas, A. V. Straube, and R. Grima, "The slow-scale linear noise approximation: An accurate, reduced stochastic description of biochemical networks under timescale separation conditions," BMC Syst. Biol. 6, 39 (2012).

${ }^{21}$ N. G. Van Kampen, Stochastic Processes in Physics and Chemistry, 3rd ed. (Elsevier, 2007).

${ }^{22}$ C. W. Gardiner, Handbook for Stochastic Methods, 2nd ed. (Springer, 1985).
${ }^{23}$ N. G. Van Kampen, “Itô versus Stratonovich," J. Stat. Phys. 24, 175-187 (1982). ${ }^{24} \mathrm{~J}$. Elf and M. Ehrenberg, "Fast evaluation of fluctuations in biochemical networks with the linear noise approximation," Genome Res. 13, 2475-2484 (2003).

${ }^{25}$ J. A. Hertz, Y. Roudi, and P. Sollich, "Path integral methods for the dynamics of stochastic and disordered systems," J. Phys. A: Math. Gen. 50, 033001 (2016).

${ }^{26}$ P. C. Martin, E. D. Siggia, and H. A. Rose, "Statistical dynamics of classical systems," Phys. Rev. A 8, 423-436 (1973).

${ }^{27} \mathrm{H}$.-K. Janssen, "Lagrangian for classical field dynamics and renormalization group calculations of dynamical critical properties," Z. Phys. B: Condens. Matter 23, 377-380 (1976).

${ }^{28} \mathrm{C}$. De Dominicis, "Dynamics as a substitute for replicas in systems with qunched random impurities," Phys. Rev. B 18, 4913-4919 (1978).

${ }^{29}$ A. Kamenev, Field Theory of Non-Equilibrium Systems (Cambridge University Press, 2011).

${ }^{30} \mathrm{~S}$. Kullback and R. A. Leibler, "On information and sufficiency," Ann. Math. Stat. 22, 79-86 (1951)

${ }^{31}$ K. Öcal, R. Grima, and G. Sanguinetti, "Parameter estimation for biochemical reaction networks using Wasserstein distances," J. Phys. A: Math. Theor. 53, 034002 (2019).

${ }^{32}$ The projection method can in principle be applied for $\varepsilon>0$ to derive reduced dynamical equations also for the fluctuating subnetwork concentrations, but the resulting expressions (e.g., for the memory functions and random force) cannot be evaluated in the closed form without further approximation. ${ }^{55,56}$

${ }^{33}$ M. Beck, A. Schmidt, J. Malmstroem, M. Claassen, A. Ori, A. Szymborska, F. Herzog, O. Rinner, J. Ellenberg, and R. Aebersold, "The quantitative proteome of a human cell line," Mol. Syst. Biol. 7, 549 (2011).

${ }^{34}$ N. Nagaraj, J. R. Wisniewski, T. Geiger, J. Cox, M. Kircher, J. Kelso, S. Pääbo, and M. Mann, "Deep proteome and transcriptome mapping of a human cancer cell line," Mol. Syst. Biol. 7, 548 (2011).

${ }^{35} \mathrm{~K}$. Iwamoto, Y. Shindo, and K. Takahashi, "Modeling cellular noise underlying heterogeneous cell responses in the epidermal growth factor signaling pathway," PLoS Comput. Biol. 12, e1005222 (2016).

${ }^{36}$ E. Kim, J.-Y. Kim, M. A. Smith, E. B. Haura, and A. R. A. Anderson, "Cell signaling heterogeneity is modulated by both cell-intrinsic and -extrinsic mechanisms: An integrated approach to understanding targeted therapy," PLoS Comput. Biol. 16, e2002930 (2018).

${ }^{37}$ B. N. Kholodenko, O. V. Demin, G. Moehren, and J. B. Hoek, "Quantification of short term signaling by the epidermal growth factor receptor," J. Biol. Chem. 274, 30169-30181 (1999).

${ }^{38}$ R. Milo, P. Jorgensen, U. Moran, G. Weber, and M. Springer, "BioNumbersthe database of key numbers in molecular and cell biology," Nucleic Acids Res. 38, D750-D753 (2010).

${ }^{39}$ C. G. Bowsher and P. S. Swain, "Identifying sources of variation and the flow of information in biochemical networks," Proc. Natl. Acad. Sci. U. S. A. 109, E1320E1328 (2012).

${ }^{40}$ A. Hilfinger and J. Paulsson, "Separating intrinsic from extrinsic fluctuations in dynamic biological systems," Proc. Natl. Acad. Sci. U. S. A. 108, 12167-12172 (2011).

${ }^{41} \mathrm{C}$. Zechner and H. Koeppl, "Uncoupled analysis of stochastic reaction networks in fluctuating environments," PLoS Comput. Biol. 10, e1003942 (2014).

${ }^{42}$ J. Falk, L. Bronstein, M. Hanst, B. Drossel, and H. Koeppl, "Context in synthetic biology: Memory effects of environments with mono-molecular reactions," J. Chem. Phys. 150, 024106 (2019).

${ }^{43}$ M. B. Elowitz, A. J. Levine, E. D. Siggia, and P. S. Swain, "Stochastic gene expression in a single cell,” Science 297, 1183-1186 (2002).

${ }^{44}$ V. Shahrezaei, J. F. Olivier, and P. S. Swain, "Colored extrinsic fluctuations and stochastic gene expression,” Mol. Syst. Biol. 4, 196 (2008).

${ }^{45} \mathrm{~T}$. Shibata and K. Fujimoto, "Noisy signal amplification in ultrasensitive signal transduction," Proc. Natl. Acad. Sci. U. S. A. 102, 331-336 (2005).

${ }^{46} \mathrm{~K}$. J. Rubin and P. Sollich, "Michaelis-Menten dynamics in protein subnetworks," J. Chem. Phys. 144, 174114 (2016). 
${ }^{47}$ E. Herrera-Delgado, R. Perez-Carrasco, J. Briscoe, and P. Sollich, "Memory functions reveal structural properties of gene regulatory networks," PLoS Comput. Biol. 14, e1006003 (2018).

${ }^{48}$ C. Zechner, J. Ruess, P. Krenn, S. Pelet, M. Peter, J. Lygeros, and H. Koeppl, "Moment-based inference predicts bimodality in transient gene expression," Proc. Natl. Acad. Sci. U. S. A. 109, 8340-8345 (2012).

${ }^{49}$ C. Zechner, M. Unger, S. Pelet, M. Peter, and H. Koeppl, "Scalable inference of heterogeneous reaction kinetics from pooled single-cell recordings," Nat. Methods 11, 197-202 (2014).

${ }^{50}$ S. D. M. Santos, P. J. Verveer, and P. I. H. Bastiaens, "Growth factor-induced MAPK network topology shapes Erk response determining PC-12 cell fate," Nat. Cell Biol. 9, 324-330 (2007).

${ }^{51}$ C. Starbuck and D. A. Lauffenburger, "Mathematical model for the effects of epidermal growth factor receptor trafficking dynamics on fibroblast proliferation responses," Biotechnol. Prog. 8, 132-143 (1992).

${ }^{52}$ M. L. Blinov, J. R. Faeder, B. Goldstein, and W. S. Hlavacek, "A network model of early events in epidermal growth factor receptor signaling that accounts for combinatorial complexity," BioSystems 83, 136-151 (2006).

${ }^{53}$ K. Rubin, "Dynamics of protein interaction subnetworks," Ph.D. thesis, King's College London, 2014.
${ }^{54}$ E. Herrera-Delgado, J. Briscoe, and P. Sollich, "Nonlinear memory functions capture and explain dynamical behaviours," arXiv:2005.04751 [physics, q-bio] (2020).

${ }^{55}$ A. J. Chorin, O. H. Hald, and R. Kupferman, "Optimal prediction and the MoriZwanzig representation of irreversible processes," Proc. Natl. Acad. Sci. U. S. A. 97, 2968-2973 (2000).

${ }^{56}$ A. Coolen and S. Rabello, "Generating functional analysis of complex formation and dissociation in large protein interaction networks," J. Phys.: Conf. Ser. 197, 012006 (2009).

${ }^{57}$ H. Kleinert, Path Integrals in Quantum Mechanics, Statistics, Polymer Physics, and Financial Markets, 4th ed. (World Scientific Publishing, 2006).

${ }^{58}$ C. Villani, Optimal Transport: Old and New, Grundlehren Der Mathematischen Wissenschaften (Springer-Verlag, Berlin, Heidelberg, 2009).

${ }^{59}$ P. Virtanen, R. Gommers, T. E. Oliphant, M. Haberland, T. Reddy, D. Cournapeau, E. Burovski, P. Peterson, W. Weckesser, J. Bright, S. J. van der Walt, M. Brett, J. Wilson, K. J. Millman, N. Mayorov, A. R. J. Nelson, E. Jones, R. Kern, E. Larson, C. J. Carey, I. Polat, Y. Feng, E. W. Moore, J. VanderPlas, D. Laxalde, J. Perktold, R. Cimrman, I. Henriksen, E. A. Quintero, C. R. Harris, A. M. Archibald, A. H. Ribeiro, F. Pedregosa, and P. van Mulbregt, "SciPy 1.0: Fundamental algorithms for scientific computing in Python,” Nat. Methods 17, 261-272 (2020). 